\title{
An efficient and rapid microwave-assisted synthesis of 1-acetyl-1H-indol-3-YL acetates
}

Article

Accepted Version

Parshotam, J. P., Brazier, J. A., Bovill, R. and Osborn, H. M. I. (2016) An efficient and rapid microwave-assisted synthesis of 1-acetyl-1H-indol-3-YL acetates. Current Microwave Chemistry, 3 (3). pp. 247-257. ISSN 2213-3364 doi:

https://doi.org/10.2174/2213335603666160108001556 Available at https://centaur.reading.ac.uk/51462/

It is advisable to refer to the publisher's version if you intend to cite from the work. See Guidance on citing.

To link to this article DOI:

http://dx.doi.org/10.2174/2213335603666160108001556

Publisher: Bentham

All outputs in CentAUR are protected by Intellectual Property Rights law, including copyright law. Copyright and IPR is retained by the creators or other copyright holders. Terms and conditions for use of this material are defined in the End User Agreement.

www.reading.ac.uk/centaur 


\section{CentAUR}

Central Archive at the University of Reading

Reading's research outputs online 
AN EFFICIENT AND RAPID MICROWAVE-ASSISTED SYNTHESIS OF 1-ACETYL-1H-INDOL-3-YL ACETATES

Jay P. Parshotam ${ }^{\mathrm{a}}$, John A. Brazier ${ }^{\mathrm{a}}$, Richard Bovill ${ }^{\mathrm{b}}$ and Helen M.I. Osborn ${ }^{* a}$,

${ }^{a}$ Reading School of Pharmacy, University of Reading, Whiteknights, Reading, RG6 6AD, UK; ${ }^{b}$ Thermofisher Scientific, Wade Road, Basingstoke, Hampshire, RG24 $8 P W, U K$

*Address correspondence to this author at the Reading School of Pharmacy, University of Reading, Whiteknights, Reading. RG6 6AD, UK; Tel/Fax: +44(0) 118 378 7338, +44(0) 118 378 6331; E-mail: h.m.i.osborn@ @rdg.ac.uk 


\section{ABSTRACT}

An efficient and rapid synthesis of 1-acetyl-1H-indol-3-yl acetate 1 and its derivatives 7 via the microwave-assisted cyclisation and decarboxylation of 2[(carboxymethyl)amino]benzoic acids $\mathbf{5}$ is described. The latter were left to react with acetic anhydride using triethylamine as the base and were subjected to microwave irradiation for 1 minute, at $80{ }^{\circ} \mathrm{C}$ with initial power of $300 \mathrm{~W}$. The target 1-acetyl-1H-indol-3-yl acetate 1 and derivatives 7 were isolated in $34-71 \%$ yield. In particular, synthesis of 1-acetyl-6-(trifluoromethyl)-1H-indol-3-yl acetate 7 and 1-acetyl-3-methyl-1H-indol-3-yl acetate 7 h is reported for the first time.

Keywords: microwave; synthesis; cyclisation; indoxyls; indoles 


\section{INTRODUCTION}

The synthesis of indoles, which are often derived from 1-acetyl-1H-indol-3-yl acetates such as $\mathbf{1}$, is of considerable synthetic interest, for example due to their antioxidant [13], antiobesity [4-5], antimalarial [6-7] and antipyretic [8] properties. Indoles have also found use in microbiology as a result of their antibacterial properties [9] and bacterial detection capabilities [10]. Thus in work conducted by Berlin et al. [10], the glycoside 5-bromo-3-indolyl- $\alpha$-L-arabinofuranoside 2 was used for the detection of $\alpha$-Larabinofuranosidase in bacterial colonies, with enzyme catalyzed hydrolysis allowing release of the aglycon 5-bromo-1H-indol-3-ol. This unstable moiety is rapidly air oxidized to the insoluble, intensely coloured 5,5'-dibromoindigo 3, leading to the formation of blue bacterial colonies (Figure 1). Different coloured indigos are formed when different halogens are present in the benzene ring of indigo; for instance, 6,6'-dichloroindigo leads to a salmon precipitate [11].

Chemical methods for the synthesis of 1-acetyl-1H-indol-3-yl acetates such as $\mathbf{1}$ are available in the literature. For example, 1 -acetyl-1H-indol-3-yl acetate and its derivatives have been furnished from 2-chlorobenzoic acids [12]. A condensation between the latter and glycine led to the 2-[(carboxymethyl)amino]benzoic acids. A final cyclisation using sodium acetate and acetic anhydride afforded the 1-acetyl-1H-indol-3-yl acetates in an overall yield range of 5-68\%. Alternatively, Guyen et al. [13] reacted 2-amino-5bromobenzoic acid with chloroacetic acid and $2 \mathrm{M} \mathrm{Na}_{2} \mathrm{CO}_{3}$ to produce 5-bromo-2-[(carboxymethyl)amino]benzoic acid. Ring closure was then achieved using acetic anhydride and sodium acetate, and 1-acetyl-5-bromo-1H-indol-3-yl acetate was obtained in an overall yield of 33\%. In a further method, Lai et al. [14] subjected 2[(carboxymethyl)amino]benzoic acid to microwave irradiation to afford 2-[acetyl(carboxymethyl)amino]benzoic acid in 92\% yield. Further microwave irradiation led to the formation of 1-acetyl-1H-indol-3-yl acetate in $86 \%$ yield. A further option has been developed by Choi et al. [15] who synthesised a range of substituted 2[(carboxymethyl)amino]benzoic acids in two steps from the respective anthranilic acids. First, the anthranilic acids were reacted with ethyl glyoxylate, sodium cyanoborohydride and $1 \%$ acetic acid to produce the 2-[(2-ethoxy-2-oxoethyl)amino]benzoic acid derivatives. The latter ester intermediates were then subjected to base hydrolysis to afford the 2-[(carboxymethyl)amino]benzoic acids in an overall yield range of $77-86 \%$. Reaction of the latter with acetic anhydride and $\mathrm{Na}_{2} \mathrm{CO}_{3}$ under reflux led to the substituted 1-acetyl-1H-indol-3-yl acetates. Whilst each of these strategies has its own advantages and weaknesses, in general the routes are often lengthy, and the 
scope of the reactions for accessing a range of indoxyl derivatives has not always been demonstrated. Thus within this paper, an alternative method for the synthesis of 1-

acetyl-1H-indol-3-yl acetates from 2-[(carboxymethyl)amino]benzoic acids is presented that is rapid, facile and efficient, and allows entry to a range of synthetically useful indoxyls.

\section{MATERIALS AND METHODS}

All chemicals used in this work were commercially available and were used without further purification. Thin layer chromatography (TLC) was performed on aluminium backed silica gel 60 plates (Merck, Germany) and spots were visualized under UV light (254 nm). Column chromatography was performed manually or by automated column chromatography. When performed manually, sand and silica $60 \AA$ (35-70 $\mu \mathrm{m})$ were used as adsorbents and these were obtained from Fisher Scientific and Fluka, respectively. When automated, a Grace Reveleris system equipped with a C18 Column (120 g) was used and the solvents were acetonitrile (HPLC grade) and 0.1\% formic acid. Analysis was monitored at the wavelengths of 210, 254 and $280 \mathrm{~nm}$. Purification in some cases was carried out by preparative high performance liquid chromatography using an Agilent 110 series with PLRP-S 100A 5 or $8 \mu \mathrm{M}$ columns, 2 x G1316A preparative pumps, a G2360A preparative autosampler and G1365B MWD detector. The mobile phases used were acetonitrile (HPLC grade) and $0.1 \%$ or $1 \%$ formic acid. The carrier gas was nitrogen. Analysis was monitored at the wavelengths of 210 and 254 nm. The microwave-assisted reactions were carried out in $10 \mathrm{~mL}$ glass vials using a Discover-S CEM (NP-1009) microwave. ${ }^{1} \mathrm{H}$ NMR spectra were recorded at 400 or $500 \mathrm{MHz}$ and ${ }^{13} \mathrm{C}$ NMR spectra were recorded at 100, 125 or $176 \mathrm{MHz}$, on a Bruker Avance DPX 400, Bruker AV II+ 500 or Bruker AV III 700 spectrometer. Chemical shifts are reported in parts per million (ppm). Coupling constants, $J$, are reported in Hertz (Hz) and peak patterns are represented as follows: s, singlet; b, broad; d, doublet; t, triplet; q, quartet; dd, doublet of doublets; ddd, doublet of doublet of doublets; m, multiplet. Infra-red (IR) spectra were recorded on a Perkin Elmer Spectrum 100 FT-IR or Perkin Elmer Spectrum RXI Spectrometer and values are reported in wavenumbers $\left(\mathrm{cm}^{-1}\right)$. Mass spectra were recorded on a Thermo Fisher Scientific LTQ Orbitrap XL mass spectrometer. Melting points were obtained using an Electrothermal digital melting point apparatus.

\subsection{Synthetic Strategy}




\subsubsection{Representative Procedure for Preparation of 2-[(Carboxymethyl)amino]benzoic acid and Derivatives (Scheme 2, Table 1)}

The respective 2-aminobenzoic acid (1 equivalent) was dissolved in $\mathrm{NaOH}(2 \mathrm{M})$. To this was added chloroacetic acid (1.2 equivalents) dissolved in $\mathrm{NaOH}$ (2M). The solution was stirred for 3 days at $80^{\circ} \mathrm{C}$. The solution was left to cool at room temperature, and addition of $\mathrm{HCl}(2 \mathrm{M})$ afforded a precipitate. An extraction with diethyl ether was carried out. Further material was retrieved by adjusting the $\mathrm{pH}$ of the aqueous solution to 3 via the addition of $\mathrm{HCl}$ (2M) and then extracting the aqueous phase with diethyl ether (x2). The combined organic fractions were dried over $\mathrm{MgSO}_{4}$, filtered and evaporated under reduced pressure. The reaction mixture was purified with the appropriate purification method.

\subsubsection{Synthesis of 2-[(carboxymethyl)amino]benzoic acid 5a}

Synthesised from 2-aminobenzoic acid (4a, $3.65 \mathrm{mmol}, 1$ equiv., $500 \mathrm{mg}$ ) and chloroacetic acid (4.38 mmol, 1.2 equiv., $413.7 \mathrm{mg})$ according to the representative proced ure described in 2.2.1. The product was purified by column chromatography on silica gel (using first $9 / 1=$ ethyl acetate/methanol and then $9 / 1 / 0.1=$ ethyl acetate/methanol/acetic acid). White/cream solid. Yield: $29 \% . R_{\mathrm{f}} 0.53$ (using 9/1/0.1= ethyl acetate/methanol/acetic acid). ${ }^{1} \mathrm{H} \mathrm{NMR}\left(\mathrm{CD}_{3} \mathrm{OD}, 400 \mathrm{MHz}, \mathrm{ppm}\right): \delta 4.01(2 \mathrm{H}, \mathrm{s}, \mathrm{CH})_{2}, 6.57-6.64(2 \mathrm{H}, \mathrm{m}$, $\operatorname{Ar}-\mathrm{H}), 7.36(1 \mathrm{H}, \mathrm{t}, J=8.0 \mathrm{~Hz}, \mathrm{Ar}-\mathrm{H}), 7.92(1 \mathrm{H}, \mathrm{d}, J=8.0 \mathrm{~Hz}, \mathrm{Ar}-\mathrm{H}) .{ }^{13} \mathrm{C}$ NMR $\left(\mathrm{CD}_{3} \mathrm{OD}, 100 \mathrm{MHz}, \mathrm{ppm}\right): \delta 45.40,112.13,112.38,116.25,133.26,135.66,151.70,171.77$, 174.11. IR (thin film): $3605,3398,2871,1675,1501,1458,741 \mathrm{~cm}^{-1}$. FTMS+ESI calculated for $\mathrm{C}_{9} \mathrm{H}_{10} \mathrm{NO}_{4}[\mathrm{M}+\mathrm{H}]^{+}: 196.0610$. Found: 196.0604 .

\subsubsection{Synthesis of 2-[(carboxymethyl)amino]-5-bromobenzoic acid 5 b}

Synthesised from 2-amino-5-bromobenzoic acid (4b, $2.33 \mathrm{mmol}, 1$ equiv., $500 \mathrm{mg})$ and chloroacetic acid (2.79 mmol, 1.2 equiv., $263.8 \mathrm{mg})$ according to the representative procedure described in 2.2.1. The product was purified by an automated Reveleris flash system equipped with a C18 $120 \mathrm{~g}$ column, flow of $85 \mathrm{~mL} / \mathrm{min}$, solvent system $=3 / 2$ formic acid $(0.1 \%)$ /acetonitrile, run length of $15 \mathrm{CV}$ and wavelenghts of 210,254 and $280 \mathrm{~nm}$. White solid. Yield: $8 \%$. $R_{\mathrm{f}} 0.59$ (using $9 / 1 / 0.1=$ ethyl acetate/methanol/acetic acid). m.p. $223-225^{\circ} \mathrm{C} .{ }^{1} \mathrm{H}$ NMR $\left(\mathrm{CD}_{3} \mathrm{OD}, 400 \mathrm{MHz}, \mathrm{ppm}\right) \delta 4.02\left(2 \mathrm{H}, \mathrm{s}, \mathrm{CH}_{2}\right), 6.57(1 \mathrm{H}, \mathrm{d}, J=8.5 \mathrm{~Hz}, \mathrm{Ar}-\mathrm{H}), 7.44(1 \mathrm{H}, \mathrm{d}, J=9.0 \mathrm{~Hz}, \mathrm{Ar}-\mathrm{H}), 7.98(1 \mathrm{H}, \mathrm{s}, \mathrm{Ar}-\mathrm{H}) .{ }^{13} \mathrm{C} \mathrm{NMR}$ 
$\left(\mathrm{CD}_{3} \mathrm{OD}, 100 \mathrm{MHz}, \mathrm{ppm}\right) \delta 45.25,107.24,113.67,114.52,135.23,138.02,150.65,170.38,173.60 . \mathrm{IR}$ (thin film): 3351, 1736, 1676, 1507, 1572, 1435, 803 cm ${ }^{-1} . \mathrm{FTMS}^{\mathrm{N}} \mathrm{ESI}$ calculated for $\mathrm{C}_{9} \mathrm{H}_{9}{ }^{79} \mathrm{BrNO}_{4}[\mathrm{M}+\mathrm{H}]^{+}:$273.9715, and calculated for $\mathrm{C}_{9} \mathrm{H}_{9}{ }^{81} \mathrm{BrNO}_{4}[\mathrm{M}+\mathrm{H}]^{+}:$275.9695. Found: 273.9711 and 275.9690 , respectively.

\subsubsection{Synthesis of 2-[(carboxymethyl)amino]-5-chlorobenzoic acid 5 c}

Synthesised from 2-amino-5-chlorobenzoic acid (4c, $2.92 \mathrm{mmol}, 1$ equiv., $500 \mathrm{mg})$ and chloroacetic acid (3.51 mmol, $1.2 \mathrm{equiv} ., 331.6 \mathrm{mg})$ according to the representative procedure described in 2.2.1. The product was purified by column chromatography on silica gel (using first $9 / 1=$ ethyl acetate/methanol and then $9 / 1 / 0.1=$ ethyl acetate/methanol/acetic acid). Cream coloured solid. Yield: $24 \% . R_{\mathrm{f}} 0.62$ (using 9/1/0.1= ethyl acetate/methanol/acetic acid). m.p. $217-219^{\circ} \mathrm{C} .{ }^{1} \mathrm{H} \mathrm{NMR}\left(\mathrm{CD}{ }_{3} \mathrm{OD}, 400 \mathrm{MHz}\right.$, ppm) $\delta 3.98\left(2 \mathrm{H}, \mathrm{s}, \mathrm{CH}_{2}\right), 6.61(1 \mathrm{H}, \mathrm{d}, J=9.0 \mathrm{~Hz}, \mathrm{Ar}-\mathrm{H}), 7.32(1 \mathrm{H}, \mathrm{dd}, J=2.0,9.0 \mathrm{~Hz}, \mathrm{Ar}-\mathrm{H}), 7.84(1 \mathrm{H}, \mathrm{d}, J=2.0 \mathrm{~Hz}, \mathrm{Ar}-\mathrm{H}) .{ }^{13} \mathrm{C} \mathrm{NMR}\left(\mathrm{CD}{ }_{3} \mathrm{OD}, 100 \mathrm{MHz}, \mathrm{ppm}\right) \delta 45.54$, 113.28, 114.11, 120.61, 132.23, 135.17, 150.34, 170.58, 173.97. IR (thin film): 3344, 1721, 1678, 1571, 1510, 1435, 808 $\mathrm{cm}^{-1}$. FTMS+ESI calculated for $\mathrm{C}_{9} \mathrm{H}_{9} \mathrm{ClNO}_{4}$ $[\mathrm{M}+\mathrm{H}]^{+}:$230.0220. Found: 230.0215 .

\subsubsection{Synthesis of 2-[(carboxymethyl)amino]-5-iodobenzoic acid $5 d$}

Synthesised from 2-amino-5-iodobenzoic acid (4d, $1.9 \mathrm{mmol}, 1$ equiv., $500 \mathrm{mg}$ ) and chloroacetic acid (2.28 mmol, 1.2 equiv., $215.6 \mathrm{mg})$ according to the representative procedure described in 2.2.1. The product was purified by column chromatography on silica gel (using first $9 / 1=$ ethyl acetate/methanol and then $9 / 1 / 0.1=$ ethyl acetate/methanol/acetic acid). Cream coloured solid. Yield: 9\%. $R_{\mathrm{f}} 0.58$ (using 9/1/0.1= ethyl acetate/methanol/acetic acid). ${ }^{1} \mathrm{H} \mathrm{NMR}\left(\mathrm{CD}{ }_{3} \mathrm{OD}, 400 \mathrm{MHz}, \mathrm{ppm}\right) \delta 3.76(2 \mathrm{H}, \mathrm{s}$, $\left.\mathrm{CH}_{2}\right), 6.41(1 \mathrm{H}, \mathrm{d}, J=9.0 \mathrm{~Hz}, \mathrm{Ar}-\mathrm{H}), 7.51(1 \mathrm{H}, \mathrm{dd}, J=2.0,9.0 \mathrm{~Hz}, \mathrm{Ar}-\mathrm{H}), 8.12(1 \mathrm{H}, \mathrm{d}, J=2.0 \mathrm{~Hz}, \mathrm{Ar}-\mathrm{H}) .{ }^{13} \mathrm{C} \mathrm{NMR}\left(\mathrm{CD} \mathrm{ND}_{3}, 176 \mathrm{MHz}, \mathrm{ppm}\right) \delta 47.32,74.22,113.59$, 121.65, 139.90, 139.92, 149.05, 174.26, 177.58. IR (thin film): $3358,1633,1606,1560,1498,1448,824 \mathrm{~cm}^{-1}$. FTMS+ESI calculated for $\mathrm{C}_{9} \mathrm{H}_{9} \mathrm{INO}_{4}[\mathrm{M}+\mathrm{H}]^{+}: 321.9576$. Found: 321.9572 .

2.2.6. Synthesis of 2-[(carboxymethyl)amino]-5-fluorobenzoic acid 5 e 
Synthesised from 2-amino-5-fluorobenzoic acid (4e, $3.23 \mathrm{mmol}, 1$ equiv., $500 \mathrm{mg}$ ) and chloroacetic acid (3.87 mmol, 1.2 equiv., $365.7 \mathrm{mg})$ according to the representative procedure described in 2.2.1. The product was purified by column chromatography on silica gel (using first $9 / 1=$ ethyl acetate/methanol and then $9 / 1 / 0.1=$ ethyl acetate/methanol/acetic acid). Light yellow solid. Yield: $18 \% . R_{\mathrm{f}} 0.78$ (using 9/1/0.1= ethyl acetate/methanol/acetic acid). ${ }^{1} \mathrm{H}$ NMR $\left(\mathrm{CD}{ }_{3} \mathrm{OD}, 400 \mathrm{MHz}, \mathrm{ppm}\right) \delta 4.00(2 \mathrm{H}, \mathrm{s}$, $\left.\mathrm{CH}_{2}\right), 6.60(1 \mathrm{H}, \mathrm{dd}, J=4.5,9.0 \mathrm{~Hz}, \mathrm{Ar}-\mathrm{H}), 7.16(1 \mathrm{H}, \mathrm{m}, \mathrm{Ar}-\mathrm{H}), 7.59(1 \mathrm{H}, \mathrm{dd}, J=3.0,9.5 \mathrm{~Hz}, \mathrm{Ar}-\mathrm{H}) .{ }^{13} \mathrm{C} \mathrm{NMR}\left(\mathrm{CD}{ }_{3} \mathrm{OD}, 100 \mathrm{MHz}, \mathrm{ppm}\right) \delta 45.62,112.30(\mathrm{~d}, J 6.5 \mathrm{~Hz})$, $113.74(\mathrm{~d}, J 7.0 \mathrm{~Hz}), 118.14(\mathrm{~d}, J 23.0 \mathrm{~Hz}), 122.82(\mathrm{~d}, J 23.0 \mathrm{~Hz}), 148.56,154.73(\mathrm{~d}, J 231.5 \mathrm{~Hz}), 170.62,173.95$. IR (thin film): $3397,1681,1569,1518,1225,810 \mathrm{~cm}{ }^{-1}$. FTMS+ESI calculated for $\mathrm{C}_{9} \mathrm{H}_{9} \mathrm{FNO}_{4}[\mathrm{M}+\mathrm{H}]^{+}:$214.0516. Found: 214.0510 .

\subsubsection{Synthesis of 2-[(carboxymethyl)amino]-4,5-difluorobenzoic acid $5 f$}

Synthesised from 2-amino-4,5-difluorobenzoic acid (4f, $2.89 \mathrm{mmol}, 1$ equiv., $500 \mathrm{mg}$ ) and chloroacetic acid (3.47 mmol, 1.2 equiv., $327.7 \mathrm{mg}$ ) according to the representative procedure described in 2.2.1. The product was purified by dry loaded column chromatography on silica gel (using first $3 / 2=$ diethyl ether/petroleum ether and then $9 / 1 / 0.1=$ ethyl acetate/methanol/acetic acid). Light yellow solid. Yield: 5\%. $R_{\mathrm{f}} 0.65$ (using 9/1/0.1= ethyl acetate/methanol/acetic acid). m.p. $215.5-221^{\circ} \mathrm{C} .{ }^{1} \mathrm{H}$ NMR $\left(\mathrm{CD}_{3} \mathrm{OD}, 400 \mathrm{MHz}, \mathrm{ppm}\right) \delta 3.99\left(2 \mathrm{H}, \mathrm{s}, \mathrm{CH}_{2}\right), 6.49(1 \mathrm{H}, \mathrm{dd}, J=6.5,13.5 \mathrm{~Hz}, \mathrm{Ar}-\mathrm{H}), 7.75(1 \mathrm{H}, \mathrm{dd}, J=9.5,1.5 \mathrm{~Hz}, \mathrm{Ar}-\mathrm{H}) .{ }^{13} \mathrm{C} \mathrm{NMR}\left(\mathrm{CD}{ }_{3} \mathrm{OD}, 100 \mathrm{MHz}, \mathrm{ppm}\right) \delta 45.54$, 100.69 (d, $J 22.0 \mathrm{~Hz}), 107.65$ (dd, $J$ 2.5, 4.5 Hz), 121.0 (dd, $J$ 3.0, $19.0 \mathrm{~Hz}), 142.03$ (dd, $J$ 13.5, 233.5 Hz), $149.90(\mathrm{~d}, J 10.0 \mathrm{~Hz}), 155.95(\mathrm{dd}, J 14.0,250.5 \mathrm{~Hz}), 170.0$, 173.45. IR (thin film): 3390, 1705, 1674, 1594, 1533, 1432, $1256 \mathrm{~cm}^{-1}$. FTMS+ESI calculated for $\mathrm{C}_{9} \mathrm{H}_{8} \mathrm{~F}_{2} \mathrm{NO}_{4}[\mathrm{M}+\mathrm{H}]^{+}: 232.0421$. Found: 232.0416.

\subsubsection{Synthesis of 2-[(carboxymethyl)amino]-4-trifluorobenzoic acid $5 g$}

Synthesised from 2-amino-4-(trifluoromethyl)benzoic acid (4g, $2.44 \mathrm{mmol}, 1$ equiv., $500 \mathrm{mg}$ ) and chloroacetic acid (2.93 mmol, 1.2 equiv., $276.5 \mathrm{mg})$ according to the representative procedure described in 2.2.1. The product was purified by column chromatography on silica gel (using first $7 / 3=$ ethyl acetate/petroleum ether and then 9/1/0.02= ethyl acetate/methanol/acetic acid). Cream coloured solid. Yield: $13 \% . R_{\mathrm{f}} 0.5$ (using 9/1/0.1= ethyl acetate/methanol/acetic acid). m.p. $220-224{ }^{\circ} \mathrm{C} .{ }^{1} \mathrm{H}$ NMR $\left(\mathrm{CD}_{3} \mathrm{OD}, 400 \mathrm{MHz}, \mathrm{ppm}\right) \delta 4.07\left(1 \mathrm{H}, \mathrm{s}, \mathrm{CH}_{2}\right), 6.82(1 \mathrm{H}, \mathrm{s}, \mathrm{Ar}-\mathrm{H}), 6.86(1 \mathrm{H}, \mathrm{d}, J=8.5 \mathrm{~Hz}, \mathrm{Ar}-\mathrm{H}), 8.07(1 \mathrm{H}, \mathrm{d}, J=8.0 \mathrm{~Hz}, \mathrm{Ar}-\mathrm{H}) .{ }^{13} \mathrm{C}$ NMR $\left(\mathrm{CD}{ }_{3} \mathrm{OD}, 100 \mathrm{MHz}, \mathrm{ppm}\right) \delta$ 
45.15, 108.86 (d, J 4.5 Hz), 111.99 (d, J 3.5 Hz), 115.0, 125.33 (d, J 271.5 Hz), 134.23, 136.61 (q, J 31.5 Hz), 151.55, 170.63, 173.40. IR (thin film): 3351, 2922, 1717, 1674,

$1581,1436,852 \mathrm{~cm}^{-1}$. FTMS+ESI calculated for $\mathrm{C}_{10} \mathrm{H}_{9} \mathrm{~F}_{3} \mathrm{NO}_{4}[\mathrm{M}+\mathrm{H}]^{+}:$264.0484. Found: 264.0479 .

\subsubsection{Representative Procedure for Preparation of 2-[(2-Ethoxy-2-oxoethyl)amino]benzoic acid and Derivatives (Scheme 3, Table 2)}

The respective 2-aminobenzoic acid (1 equivalent) was left to stir in methanol, acetic acid (0.74 equivalents) and ethyl glyoxylate (1.5 equivalents, $50 \%$ in toluene) for 2.25 hours at room temperature. Then $\mathrm{NaBH}_{3}(\mathrm{CN})$ (1.5 equivalents) was added and the reaction was left to stir for an additional 1.75 hours at room temperature. The solvent was evaporated under reduced pressure and the residue dissolved in ethyl acetate. This was then washed twice with saturated $\mathrm{NH}_{4} \mathrm{Cl}$. $\mathrm{The}_{\mathrm{s}}$ organic layer was dried over $\mathrm{MgSO}_{4}$, filtered and evaporated under reduced pressure. The reaction mixture was purified with the appropriate purification method.

\subsubsection{Synthesis of 2-[(2-ethoxy-2-oxoethyl)amino]benzoic acid 6 a}

Synthesised from 2-aminobenzoic acid (4a, 7.3 mmol, 1 equiv., 1 g), acetic acid (5.4 mmol, 0.74 equiv., $308.8 \mu \mathrm{L})$, ethyl glyoxylate (10.95 mmol, 1.5 equiv., 2.17 mL) and $\mathrm{NaBH}_{3}(\mathrm{CN})(10.95 \mathrm{mmol}, 1.5$ equiv., $687.8 \mathrm{mg})$ according to the representative procedure described in 2.2.9. Product 6a was used for the next step without purification.

\subsubsection{Synthesis of 5-bromo-2-[(2-ethoxy-2-oxoethyl)amino]benzoic acid $6 b$}

Synthesised from 2-amino-5-bromobenzoic acid (4b, 4.65 mmol, 1 equiv., 1 g), acetic acid (3.44 mmol, 0.74 equiv., $196.9 \mu \mathrm{L})$, ethyl glyoxylate (6.98 mmol, 1.5 equiv., $1.383 \mathrm{~mL})$ and $\mathrm{NaBH}_{3}(\mathrm{CN})(6.98 \mathrm{mmol}, 1.5$ equiv., $438.5 \mathrm{mg})$ according to the representative procedure described in 2.2 .9 . The product was purified by column chromatography (using 7/3=ethyl acetate/petroleum ether). White solid. Yield: $89 \% . R_{\mathrm{f}} 0.63$ (using $7 / 3=$ ethyl acetate/petroleum ether). ${ }^{1} \mathrm{H} \mathrm{NMR}(\mathrm{CDCl} 3,500 \mathrm{MHz}$, ppm) $\delta$ $1.31\left(3 \mathrm{H}, \mathrm{t}, J=7.0 \mathrm{~Hz}, \mathrm{CH}_{3}\right), 3.99\left(2 \mathrm{H}, \mathrm{s}, \mathrm{CH}_{2}\right), 4.27\left(2 \mathrm{H}, \mathrm{q}, J=7.0 \mathrm{~Hz}, \mathrm{CH}_{2}\right), 6.44(1 \mathrm{H}, \mathrm{d}, J=9.0 \mathrm{~Hz}, \mathrm{Ar}-\mathrm{H}), 7.46(1 \mathrm{H}, \mathrm{d}, J=8.0 \mathrm{~Hz}, \mathrm{Ar}-\mathrm{H}), 8.10(1 \mathrm{H}, \mathrm{s}, \mathrm{Ar}-\mathrm{H}) .{ }^{13} \mathrm{C} \mathrm{NMR}$ $\left(\mathrm{CDCl}_{3}, 125 \mathrm{MHz}, \mathrm{ppm}\right) \delta 14.19,44.98,61.66,107.13,111.22,113.19,134.89,138.18,149.39,169.93,172.34$. IR (thin film): 3336, 1734, 1671, 1566, 1508, 1454, 804, 679 $\mathrm{cm}^{-1}$. FTMS+ESI calculated for $\mathrm{C}_{11} \mathrm{H}_{13}{ }^{79} \mathrm{BrNO}_{4}[\mathrm{M}+\mathrm{H}]^{+}: 302.0022$, and calculated for $\mathrm{C}_{11} \mathrm{H}_{13}{ }^{81} \mathrm{BrNO}_{4}[\mathrm{M}+\mathrm{H}]^{+}: 304.0002$. Found: 302.0026 and 304.0003 , respectively. 


\subsubsection{Synthesis of 5-chloro-2-[(2-ethoxy-2-oxoethyl)amino]benzoic acid 6c}

Synthesised from 2-amino-5-chlorobenzoic acid (4c, 5.85 mmol, 1 equiv., 1 g), acetic acid (4.33 mmol, 0.74 equiv., $247.5 \mu \mathrm{L})$, ethyl glyoxylate (8.77 mmol, 1.5 equiv., 1.739 $\mathrm{mL})$ and $\mathrm{NaBH}_{3}(\mathrm{CN})(8.77 \mathrm{mmol}, 1.5$ equiv., $551.2 \mathrm{mg})$ according to the representative procedure described in 2.2 .9 . The product was purified by column chromatography (using $7 / 3=$ ethyl acetate/petroleum ether). Cream coloured solid. Yield: $80 \% . R_{\mathrm{f}} 0.32$ (using $7 / 3=$ ethyl acetate/petroleum ether). m.p. $145-147^{\circ} \mathrm{C} .{ }^{1} \mathrm{H} \mathrm{NMR}\left(\mathrm{CDCl}{ }_{3}\right.$, $400 \mathrm{MHz}$, ppm) $\delta 1.31\left(3 \mathrm{H}, \mathrm{t}, J=7.0 \mathrm{~Hz}, \mathrm{CH}_{3}\right), 4.00\left(2 \mathrm{H}, \mathrm{s}, \mathrm{CH}_{2}\right), 4.27\left(2 \mathrm{H}, \mathrm{q}, J=7.0 \mathrm{~Hz}, \mathrm{CH}_{2}\right), 6.49(1 \mathrm{H}, \mathrm{d}, J=9.0 \mathrm{~Hz}, \mathrm{Ar}-\mathrm{H}), 7.34(1 \mathrm{H}, \mathrm{dd}, J=2.5,9.0 \mathrm{~Hz}, \mathrm{Ar}-\mathrm{H}), 7.96(1 \mathrm{H}, \mathrm{d}, J=$ 2.5 Hz Ar-H), 8.07 (1H, b, -NH-). ${ }^{13} \mathrm{C}$ NMR ( $\left.\mathrm{CDCl}_{3}, 100 \mathrm{MHz}, \mathrm{ppm}\right) \delta$ 14.18, 45.03, 61.65, 110.63, 112.81, 120.44, 131.93, 135.50, 149.06, 169.99, 172.59. IR (thin film): $3352,1739,1673,1567,1511,1452,809 \mathrm{~cm}^{-1}$. FTMS+ESI calculated for $\mathrm{C}_{11} \mathrm{H}_{13}{ }^{35} \mathrm{ClNO}_{4}[\mathrm{M}+\mathrm{H}]^{+}:$258.0528, and calculated for $\mathrm{C}_{11} \mathrm{H}_{13}{ }^{37} \mathrm{ClNO}{ }_{4}[\mathrm{M}+\mathrm{H}]^{+}: 260.0498$. Found: 258.0528 and 258.0497 , respectively.

\subsubsection{Synthesis of 5-iodo-2-[(2-ethoxy-2-oxoethyl)amino]benzoic acid $6 d$}

Synthesised from 2-amino-5-iodobenzoic acid (4d, 3.8 mmol, 1 equiv., 1 g), acetic acid (2.81 mmol, 0.74 equiv., $161 \mu \mathrm{L})$, ethyl glyoxylate (5.7 mmol, 1.5 equiv., 1.131 mL) and $\mathrm{NaBH}_{3}(\mathrm{CN})(5.7 \mathrm{mmol}, 1.5$ equiv., $358.5 \mathrm{mg}$ ) according to the representative procedure described in 2.2.9. The product was purified by column chromatography (using 1/1=ethyl acetate/petroleum ether). Cream coloured solid. Yield: $90 \% . R_{\mathrm{f}} 0.31$ (using 1/1=ethyl acetate/petroleum ether). m.p. $169-173.5^{\circ} \mathrm{C} .{ }^{1} \mathrm{H}$ NMR $\left(\mathrm{CD}{ }_{3} \mathrm{OD}, 400 \mathrm{MHz}\right.$, ppm) $\delta 1.28\left(3 \mathrm{H}, \mathrm{t}, J=7.0 \mathrm{~Hz}, \mathrm{CH}_{3}\right), 4.04\left(2 \mathrm{H}, \mathrm{s}, \mathrm{CH}_{2}\right), 4.23\left(2 \mathrm{H}, \mathrm{q}, J=7.0 \mathrm{~Hz}, \mathrm{CH}_{2}\right), 6.44(1 \mathrm{H}, \mathrm{d}, J=9.0 \mathrm{~Hz}, \mathrm{Ar}-\mathrm{H}), 7.58(1 \mathrm{H}, \mathrm{dd}, J=2.0,9.0 \mathrm{~Hz}, \mathrm{Ar}-\mathrm{H}), 8.15(1 \mathrm{H}, \mathrm{d}, J=$ $2.0 \mathrm{~Hz}, \mathrm{Ar}-\mathrm{H}) .{ }^{13} \mathrm{C}$ NMR $\left(\mathrm{CD}_{3} \mathrm{OD}, 100 \mathrm{MHz}, \mathrm{ppm}\right) \delta 14.50,45.43,62.39,75.54,114.58,114.95,141.37,143.68,151.09,170.30,172.08 . \mathrm{IR}($ thin film): 3326, 2957, 2922, 2851, 1731, 1670, 1606, 1563, 1503, $805 \mathrm{~cm}^{-1}$. FTMS+ESI calculated for $\mathrm{C}_{11} \mathrm{H}_{13} \mathrm{INO}_{4}[\mathrm{M}+\mathrm{H}]^{+}: 349.9889$. Found: 349.9884 .

\subsubsection{Synthesis of 5-fluoro-2-[(2-ethoxy-2-oxoethyl)amino]benzoic acid 6e}

Synthesised from 2-amino-5-fluorobenzoic acid (4e, 6.45 mmol, 1 equiv., 1 g), acetic acid (4.77 mmol, 0.74 equiv., $273 \mu \mathrm{L})$, ethyl glyoxylate (9.68 mmol, 1.5 equiv., 1.918 $\mathrm{mL})$ and $\mathrm{NaBH}_{3}(\mathrm{CN})(9.68 \mathrm{mmol}, 1.5$ equiv., $608 \mathrm{mg}$ ) according to the representative procedure described in 2.2 .9 . The product was purified by column chromatography 
(using 7/3=ethyl acetate/petroleum ether). Cream coloured solid. Yield: 83\%. $R_{\mathrm{f}} 0.69$ (using 7/3=ethyl acetate/petroleum ether). m.p. $166-168^{\circ} \mathrm{C} .{ }^{1} \mathrm{H}$ NMR $\left(\mathrm{CD}_{3} \mathrm{OD}\right.$, 400 MHz, ppm) $\delta 1.30\left(3 \mathrm{H}, \mathrm{t}, J=7.0 \mathrm{~Hz}, \mathrm{CH}_{3}\right), 4.07\left(2 \mathrm{H}, \mathrm{s}, \mathrm{CH}_{2}\right), 4.24\left(2 \mathrm{H}, \mathrm{q}, J=7.0 \mathrm{~Hz}, \mathrm{CH}_{2}\right), 6.62(1 \mathrm{H}, \mathrm{dd}, J=4.5,9.0 \mathrm{~Hz}, \mathrm{Ar}-\mathrm{H}), 7.17(1 \mathrm{H}, \mathrm{ddd}, J=3.0,8.0,11.0 \mathrm{~Hz}, \mathrm{Ar}-$ $\mathrm{H}), 7.61(1 \mathrm{H}, \mathrm{dd}, J=3.0,9.5 \mathrm{~Hz}, \mathrm{Ar}-\mathrm{H}) .{ }^{13} \mathrm{C} \mathrm{NMR}\left(\mathrm{CD}_{3} \mathrm{OD}, 100 \mathrm{MHz}, \mathrm{ppm}\right) \delta 14.49,45.91,62.30,112.52,113.75(\mathrm{~d}, J 7.0 \mathrm{~Hz}), 118.16(\mathrm{~d}, J 23.0 \mathrm{~Hz}), 122.77(\mathrm{~d}, J 23.0$ $\mathrm{Hz}), 148.56,154.81$ (d, $J 231.5 \mathrm{~Hz}), 170.62,172.42$. IR (thin film): 1734, 1670, 1558, 1429, $805 \mathrm{~cm}^{-1}$. FTMS+ESI calculated for $\mathrm{C}_{11} \mathrm{H}_{13} \mathrm{FNO}_{4}[\mathrm{M}+\mathrm{H}]^{+}: 242.0829$. Found: 242.0822 .

\subsubsection{Synthesis of 4,5-fluoro-2-[(2-ethoxy-2-oxoethyl)amino]benzoic acid 6f}

Synthesised from 2-amino-4,5-difluorobenzoic acid (4f, $5.78 \mathrm{mmol}, 1$ equiv., $1 \mathrm{~g}$ ), acetic acid (4.28 mmol, 0.74 equiv., $244.6 \mu \mathrm{L})$, ethyl glyoxylate ( $8.67 \mathrm{mmol}, 1.5$ equiv., $1.718 \mathrm{~mL})$ and $\mathrm{NaBH}_{3}(\mathrm{CN})(8.7 \mathrm{mmol}, 1.5$ equiv., $544.8 \mathrm{mg}$ ) according to the representative procedure described in 2.2.9. The product was purified by column chromatography (using 7/3=ethyl acetate/petroleum ether). White solid. Yield: $82 \% . R_{\mathrm{f}} 0.46$ (using $7 / 3=$ ethyl acetate/petroleum ether). m.p. $175-178^{\circ} \mathrm{C} .{ }^{1} \mathrm{H} \mathrm{NMR}\left(\mathrm{CD}{ }_{3} \mathrm{OD}\right.$, $400 \mathrm{MHz}, \mathrm{ppm}) \delta 1.29\left(3 \mathrm{H}, \mathrm{t}, J=7.0 \mathrm{~Hz}, \mathrm{CH}_{3}\right), 4.03\left(2 \mathrm{H}, \mathrm{s}, \mathrm{CH}_{2}\right), 4.23\left(2 \mathrm{H}, \mathrm{q}, J=7.0 \mathrm{~Hz}, \mathrm{CH}_{2}\right), 6.49(1 \mathrm{H}, \mathrm{dd}, J=6.5,13.5 \mathrm{~Hz}, \mathrm{Ar}-\mathrm{H}), 7.75(1 \mathrm{H}, \mathrm{dd}, J=9.5,11.5 \mathrm{~Hz}, \mathrm{Ar}-$ H). ${ }^{13} \mathrm{C}$ NMR $\left(\mathrm{CD}_{3} \mathrm{OD}, 100 \mathrm{MHz}, \mathrm{ppm}\right) \delta$ 14.48, 45.79, 62.42, 100.76 (d, $\left.J 21.0 \mathrm{~Hz}\right), 107.84,121.03$ (d, $\left.J 19.0 \mathrm{~Hz}\right), 142.10(\mathrm{dd}, J 14.0,234.0 \mathrm{~Hz}), 149.91(\mathrm{~d}, J 11.0 \mathrm{~Hz})$, 155.92 (dd, $J$ 14.0, $249.0 \mathrm{~Hz}$ ), 170.02, 171.93. IR (thin film): 1734, 1681, 1585, 1535, $1429 \mathrm{~cm}^{-1}$. FTMS+ESI calculated for $\mathrm{C}_{11} \mathrm{H}_{12} \mathrm{~F}_{2} \mathrm{NO}_{4}[\mathrm{M}+\mathrm{H}]^{+}: 260.0734$. Found: 260.0729 .

\subsubsection{Synthesis of 4-trifluoro-2-[(2-ethoxy-2-oxoethyl)amino]benzoic acid $6 \mathrm{~g}$}

Synthesised from 2-amino-4-(trifluoromethyl)benzoic acid (4g, 4.88 mmol, 1 equiv., 1 g), acetic acid (3.61 mmol, 0.74 equiv., $206.4 \mu \mathrm{L})$, ethyl glyoxylate (7.32 mmol, 1.5 equiv., $1.45 \mathrm{~mL})$ and $\mathrm{NaBH}_{3}(\mathrm{CN})(7.32 \mathrm{mmol}, 1.5$ equiv., $459.7 \mathrm{mg})$ according to the representative procedure described in 2.2.9. Product $\mathbf{6 g}$ was used for the next step without purification.

2.2.17. Synthesis of 5-methyl-2-[(2-ethoxy-2-oxoethyl)amino]benzoic acid $6 \mathrm{~h}$ 
Synthesised from 2-amino-5-methylbenzoic acid (4h, 6.62 mmol, 1 equiv., 1 g), acetic acid (4.9 mmol, 0.74 equiv., $280.2 \mu \mathrm{L})$, ethyl glyoxylate (9.93 mmol, 1.5 equiv., 1.968 $\mathrm{mL})$ and $\mathrm{NaBH}_{3}(\mathrm{CN})(9.93 \mathrm{mmol}, 1.5$ equiv., $624 \mathrm{mg})$ according to the representative procedure described in 2.2.9. The product was purified by dry loaded column chromatography (using 3/2=ethyl acetate/petroleum ether). White solid. Yield: $83 \% . R_{\mathrm{f}} 0.74$ (using $3 / 2=$ ethyl acetate/petroleum ether). m.p. $151.1-152.2^{\circ} \mathrm{C} .{ }^{1} \mathrm{H}$ NMR $\left(\mathrm{CDCl}{ }_{3}\right.$, $400 \mathrm{MHz} . \mathrm{ppm}) \delta 1.31\left(3 \mathrm{H}, \mathrm{t}, J=7.0 \mathrm{~Hz}, \mathrm{CH}_{3}\right), 2.25\left(3 \mathrm{H}, \mathrm{s}, \mathrm{CH}_{3}\right), 4.01\left(2 \mathrm{H}, \mathrm{s}, \mathrm{CH}_{2}\right), 4.26\left(2 \mathrm{H}, \mathrm{q}, J=7.0 \mathrm{~Hz}, \mathrm{CH}_{2}\right), 6.47(1 \mathrm{H}, \mathrm{d}, J=8.5 \mathrm{~Hz}, \mathrm{Ar}-\mathrm{H}), 7.23(1 \mathrm{H}, \mathrm{dd}, J=2.0$, 8.5 Hz, Ar-H), $7.82(1 \mathrm{H}, \mathrm{d}, J=1.5 \mathrm{~Hz}, \mathrm{Ar}-\mathrm{H}) .{ }^{13} \mathrm{C} \mathrm{NMR}\left(\mathrm{CDCl}_{3}, 100 \mathrm{MHz}, \mathrm{ppm}\right) \delta$ 14.20, 20.14, 45.24, 61.39, 109.66, 111.43, 124.83, 132.57, 136.69, 148.62, 170.51, 173.72. IR (thin film): $3348,1735,1670,1571,1524,802 \mathrm{~cm}^{-1}$. FTMS+ESI calculated for $\mathrm{C}_{12} \mathrm{H}_{16} \mathrm{NO}_{4}[\mathrm{M}+\mathrm{H}]^{+}: 238.1079$. Found: 238.1075 .

\subsubsection{Synthesis of 3-methyl-2-[(2-ethoxy-2-oxoethyl)amino]benzoic acid $6 i$}

Synthesised from 2-amino-3-methylbenzoic acid (4i, 6.62 mmol, 1 equiv., 1 g), acetic acid (4.9 mmol, 0.74 equiv., $280.2 \mu \mathrm{L})$, ethyl glyoxylate (9.93 mmol, 1.5 equiv., 1.968 $\mathrm{mL})$ and $\mathrm{NaBH}_{3}(\mathrm{CN})(9.93 \mathrm{mmol}, 1.5$ equiv., $624 \mathrm{mg})$ according to the representative procedure described in 2.2.9. Product $\mathbf{6} \mathbf{i}$ was used for the next step without purification.

\subsubsection{Representative Procedure for Preparation of 2-[(Carboxymethyl)amino]benzoic acid and Derivatives (Scheme 3, Table 2)}

The respective 2-[(2-ethoxy-2-oxoethyl)amino]benzoic acid was left to react in methanol and $\mathrm{NaOH}$ (1M) for 3 hours. Precipitation with $\mathrm{HCl}$ (1M) afforded the final desired product with characterization data as provided before.

\subsubsection{Synthesis of 2-[(carboxymethyl)amino]benzoic acid 5 a}

Synthesised from impure 2-[(2-ethoxy-2-oxoethyl)amino]benzoic acid (6a) according to the representative procedure described in 2.2.19. Overall yield: 96\%. Data was as presented previously in 2.2 .2

2.2.21. Synthesis of 2-[(carboxymethyl)amino]-5-bromobenzoic acid 5 b 
Synthesised from 5-bromo-2-[(2-ethoxy-2-oxoethyl)amino]benzoic acid (6b, 1.66 mmoles, $500 \mathrm{mg})$ according to the representative procedure described in 2.2.19. Yield: $90 \%$. Data was as presented previously in 2.2 .3

\subsubsection{Synthesis of 2-[(carboxymethyl)amino]-5-chlorobenzoic acid 5c}

Synthesised from 5-chloro-2-[(2-ethoxy-2-oxoethyl)amino]benzoic acid (6c, 1.95 mmoles, $500 \mathrm{mg})$ according to the representative procedure described in 2.2 .19 . Yield: 91\%. Data was as presented previously in 2.2 .4

\subsubsection{Synthesis of 2-[(carboxymethyl)amino]-5-iodobenzoic acid 5d}

Synthesised from 5-iodo-2-[(2-ethoxy-2-oxoethyl)amino]benzoic acid (6d, 1.43 mmoles, $500 \mathrm{mg})$ according to the representative procedure described in 2.2.19. Yield: 67\%.

Data was as presented previously in 2.2 .5

\subsubsection{Synthesis of 2-[(carboxymethyl)amino]-5-fluorobenzoic acid 5e}

Synthesised from 5-fluoro-2-[(2-ethoxy-2-oxoethyl)amino]benzoic acid (6e, 2.07 mmoles, $500 \mathrm{mg})$ according to the representative procedure described in 2.2.19. Yield: $89 \%$.

Data was as presented previously in 2.2.6

\subsubsection{Synthesis of 2-[(carboxymethyl)amino]-4,5-difluorobenzoic acid $5 f$}

Synthesised from 4,5-difluoro-2-[(2-ethoxy-2-oxoethyl)amino]benzoic acid (6f, 1.93 mmoles, $500 \mathrm{mg}$ ) according to the representative procedure described in 2.2.19. Yield: $76 \%$. Data was as presented previously in 2.2 .7

\subsubsection{Synthesis of 2-[(carboxymethyl)amino]-4-trifluorobenzoic acid $5 g$}


Synthesised from impure 4-trifluoro-2-[(2-ethoxy-2-oxoethyl)amino]benzoic acid (6g) according to the representative procedure described in 2.2.19. Overall yield: 52\%. Data was as presented previously in 2.2 .8

\subsubsection{Synthesis of 2-[(carboxymethyl)amino]-5-methylbenzoic acid 5 h}

Synthesised from 5-methyl-2-[(2-ethoxy-2-oxoethyl)amino]benzoic acid (6h, 2.1 mmoles, $0.5 \mathrm{~g})$ according to the representative procedure described in 2.2 .19 . White solid. Yield: $100 \% . R_{\mathrm{f}} 0.64$ (using 9/1/0.1=ethyl acetate/methanol/acetic acid). m.p. $210-212^{\circ} \mathrm{C} .{ }^{1} \mathrm{H}$ NMR $\left(\mathrm{CD}_{3} \mathrm{OD}, 400 \mathrm{MHz}, \mathrm{ppm}\right) \delta 2.22(3 \mathrm{H}, \mathrm{s}, \mathrm{CH}), 3.99\left(2 \mathrm{H}, \mathrm{s}, \mathrm{CH}_{2}\right), 6.53$ $(1 \mathrm{H}, \mathrm{d}, J=8.5 \mathrm{~Hz}, \mathrm{Ar}-\mathrm{H}), 7.20(1 \mathrm{H}, \mathrm{d}, J=8.5 \mathrm{~Hz}, \mathrm{Ar}-\mathrm{H}), 7.73(1 \mathrm{H}, \mathrm{s}, \mathrm{Ar}-\mathrm{H}) .{ }^{13} \mathrm{C} \mathrm{NMR}\left(\mathrm{CD}_{3} \mathrm{OD}, 100 \mathrm{MHz}, \mathrm{ppm}\right) \delta 20.25,45.55,112.05,112.56,125.40,133.08,136.52$, 149.79, 171.83, 174.20. IR (thin film): 3373, 1713, 1633, 1599, 1571, 1435, $1397 \mathrm{~cm}^{-1}$. FTMS+ESI calculated for $\mathrm{C}_{10} \mathrm{H}_{12} \mathrm{NO}_{4}[\mathrm{M}+\mathrm{H}]^{+}: 210.0766$. Found: 210.0759.

\subsubsection{Synthesis of 2-[(carboxymethyl)amino]-3-methylbenzoic acid 5i}

Synthesised from 3-methyl-2-[(2-ethoxy-2-oxoethyl)amino]benzoic acid (6i) according to the representative procedure described in 2.2.19. The product was purified by preparative HPLC using a PLRP-S $100 \AA 8 \mu \mathrm{m}$ column, flow of $72 \mathrm{~mL} / \mathrm{min}$, solvent system 9/1 formic acid (1\%)/acetonitrile and wavelength of 210 nm. Golden rod crystalline solid. Overall yield: $6 \%$. m.p. $168-172^{\circ} \mathrm{C} .{ }^{1} \mathrm{H}$ NMR $\left(\mathrm{D}_{2} \mathrm{O}, 400 \mathrm{MHz}, \mathrm{ppm}\right) \delta 2.29\left(3 \mathrm{H}, \mathrm{s}, \mathrm{CH}_{3}\right), 3.67(2 \mathrm{H}, \mathrm{s}, \mathrm{CH}), 7.20(1 \mathrm{H}, \mathrm{t}, J=7.5 \mathrm{~Hz}, \mathrm{Ar}-\mathrm{H}), 7.34(1 \mathrm{H}, \mathrm{d}, J=$ $7.5 \mathrm{~Hz}, \mathrm{Ar}-\mathrm{H}), 7.69(1 \mathrm{H}, \mathrm{d}, J=7.5 \mathrm{~Hz}, \mathrm{Ar}-\mathrm{H}) .{ }^{13} \mathrm{C}$ NMR $\left(\mathrm{D}_{2} \mathrm{O}, 100 \mathrm{MHz}, \mathrm{ppm}\right) \delta$ 16.64, 51.50, 126.81, 127.25, 128.89, 131.70, 134.86, 137.72, 173.41, 173.49. FTMS+ESI calculated for $\mathrm{C}_{10} \mathrm{H}_{12} \mathrm{NO}_{4}[\mathrm{M}+\mathrm{H}]^{+}:$210.0766. Found: 210.0760 .

\subsubsection{Representative Procedure for Preparation of 1-Acetyl-1H-indol-3-yl acetate and Derivatives (Scheme 4, Table 3)}

In a microwave tube, the reaction mixture containing the respective 2-[(carboxymethyl)amino]benzoic acid (1 equivalent), acetic anhydride (59 equivalents) and triethylamine ( 3 equivalents), was subjected to sonication for 0.25 minutes for uniform mixing and was then irradiated by microwave at an initial power of $300 \mathrm{~W}$ for 1 minute 
at $80^{\circ} \mathrm{C}$. The respective organic solvent was added to the mixture and this was washed twice with saturated $\mathrm{NaHCO}_{3}$. The organic layer was washed with water, dried over $\mathrm{MgSO}_{4}$, filtered and evaporated under reduced pressure. Purification using the appropriate purification technique furnished the desired 1-acetyl-1H-indol-3-yl acetate.

\subsubsection{Synthesis of 1-acetyl-1H-indol-3-yl acetate 1}

Synthesised from 2-[(carboxymethyl)amino]benzoic acid (5a, 0.51 mmoles, $100 \mathrm{mg})$, acetic anhydride (30.25 mmoles, $2.854 \mathrm{~mL})$ and triethylamine $(1.54 \mathrm{mmoles}, 214.5 \mu \mathrm{L})$ according to the representative procedure described in 2.2.29. Extractions were carried out with ethyl acetate. The product was purified by column chromatography (using $3 / 2=$ ethyl acetate/petroleum ether). Orange solid. Yield: $40 \% . R_{\mathrm{f}} 0.83$ (using $3 / 2$ ethyl acetate/petroleum ether). ${ }^{1} \mathrm{H} \mathrm{NMR}\left(\mathrm{CDCl} \mathrm{l}_{3}, 400 \mathrm{MHz}, \mathrm{ppm}\right) \delta 2.39\left(3 \mathrm{H}, \mathrm{s}, \mathrm{CH}_{3} \mathrm{COO}\right)$, $2.62\left(3 \mathrm{H}, \mathrm{s}, \mathrm{CH}_{3} \mathrm{CON}\right), 7.31(1 \mathrm{H}, \mathrm{t}, J=8.0 \mathrm{~Hz}, \mathrm{Ar}-\mathrm{H}), 7.40(1 \mathrm{H}, \mathrm{t}, J=8.0 \mathrm{~Hz}, \mathrm{Ar}-\mathrm{H}), 7.55(1 \mathrm{H}, \mathrm{d}, J=8.0 \mathrm{~Hz}, \mathrm{Ar}-\mathrm{H}), 7.72(1 \mathrm{H}, \mathrm{s}, \mathrm{Ar}-\mathrm{H}), 8.47(1 \mathrm{H}, \mathrm{d}, J=8.0 \mathrm{~Hz}, \mathrm{Ar}-\mathrm{H}) .{ }^{13} \mathrm{C}$ $\mathrm{NMR}\left(\mathrm{CDCl}_{3}, 100 \mathrm{MHz}, \mathrm{ppm}\right) \delta 21.06,23.94,113.31,116.70,117.47,123.59,123.76,126.22,132.88,134.64,167.88,168.74$. IR (thin film): 1739, 1693, 1602, 1440, 1381, $733 \mathrm{~cm}^{-1}$. FTMS+ESI calculated for $\mathrm{C}_{12} \mathrm{H}_{11} \mathrm{NO}_{3}[\mathrm{M}+\mathrm{H}]^{+}:$218.0817. Found: 218.0812 .

\subsubsection{Synthesis of 1-acetyl-5-bromo-1H-indol-3-yl acetate 7 a}

Synthesised from 2-[(carboxymethyl)amino]-5-bromobenzoic acid $(\mathbf{5 b}, 0.92 \mathrm{mmoles}, 250 \mathrm{mg})$, acetic anhydride $(54 \mathrm{mmoles}, 5.099 \mathrm{~mL})$ and triethylamine $(2.75 \mathrm{mmoles}$, 383.2 $\mu \mathrm{L}$ ) according to the representative procedure described in 2.2.29. Extractions were carried out with ethyl acetate. The product was purified by column chromatography (using $3 / 2=$ petroleum ether/ethyl acetate). Light yellow solid. Yield: $59 \%$. $R_{\mathrm{f}} 0.73$ (using 3/2 petroleum ether/ethyl acetate). m.p. $121-124{ }^{\circ} \mathrm{C} .{ }^{1} \mathrm{H} \mathrm{NMR}\left(\mathrm{CDCl} \mathrm{H}_{3}, 400 \mathrm{MHz}\right.$, ppm) $\delta 2.39\left(3 \mathrm{H}, \mathrm{s}, \mathrm{CH}_{3} \mathrm{COO}\right), 2.61\left(3 \mathrm{H}, \mathrm{s}, \mathrm{CH}_{3} \mathrm{CON}\right), 7.48(1 \mathrm{H}, \mathrm{dd}, J=9.0,1.5 \mathrm{~Hz}, \mathrm{Ar}-\mathrm{H}), 7.69(1 \mathrm{H}, \mathrm{d}, J=1.5 \mathrm{~Hz}, \mathrm{Ar}-\mathrm{H}), 7.73(1 \mathrm{H}, \mathrm{s}, \mathrm{Ar}-\mathrm{H}), 8.35(1 \mathrm{H}, \mathrm{d}, J=9.0 \mathrm{~Hz}, \mathrm{Ar}-$ H). ${ }^{13} \mathrm{C} \mathrm{NMR}\left(\mathrm{CDCl}_{3}, 100 \mathrm{MHz}, \mathrm{ppm}\right) \delta 21.01,23.79,114.34,117.10,118.19,120.31,125.20,129.08,131.46,133.54,167.68,168.60$. IR (thin film): 1757, 1707, 1592, 1568, $1445 \mathrm{~cm}^{-1}$. FTMS+ESI calculated for $\mathrm{C}_{12} \mathrm{H}_{11}{ }^{79} \mathrm{BrNO}_{3}[\mathrm{M}+\mathrm{H}]^{+}:$295.9923, and calculated for $\mathrm{C}_{12} \mathrm{H}_{11}{ }^{81} \mathrm{BrNO}_{3}[\mathrm{M}+\mathrm{H}]^{+}: 297.9902$. Found: 295.9919 and 297.9897 , respectively.

2.2.32. Synthesis of 1-acetyl-5-chloro-1H-indol-3-yl acetate $7 b$ 
Synthesised from 2-[(carboxymethyl)amino]-5-chlorobenzoic acid (5c, 0.79 mmoles, $180 \mathrm{mg})$, acetic anhydride (46.37 mmoles, $4.375 \mathrm{~mL})$ and triethylamine (2.36 mmoles, $328.9 \mu \mathrm{L}$ ) according to the representative procedure described in 2.2.29. Extractions were carried out with dichloromethane. The product was purified by column chromatography (using 3/2= petroleum ether/ethyl acetate). Light green solid. Yield: $61 \% . R_{\mathrm{f}} 0.56$ (using $3 / 2$ petroleum ether/ethyl acetate). ${ }^{1} \mathrm{H} \mathrm{NMR}\left(\mathrm{CDCl}{ }_{3}\right.$, $400 \mathrm{MHz}$, ppm) $\delta 2.38\left(3 \mathrm{H}, \mathrm{s}, \mathrm{CH}_{3} \mathrm{COO}\right), 2.60\left(3 \mathrm{H}, \mathrm{s}, \mathrm{CH}_{3} \mathrm{CON}\right), 7.33(1 \mathrm{H}, \mathrm{dd}, J=2.0,9.0 \mathrm{~Hz}, \mathrm{Ar}-\mathrm{H}), 7.50(1 \mathrm{H}, \mathrm{d}, J=2.0 \mathrm{~Hz}, \mathrm{Ar}-\mathrm{H}), 7.73(1 \mathrm{H}, \mathrm{s}, \mathrm{Ar}-\mathrm{H}), 8.38(1 \mathrm{H}, \mathrm{d}, J=9.0 \mathrm{~Hz}, \mathrm{Ar}-$ H). ${ }^{13} \mathrm{C} \mathrm{NMR}\left(\mathrm{CDCl}_{3}, 100 \mathrm{MHz}, \mathrm{ppm}\right) \delta 21.0,23.74,114.49,117.26,117.85,124.75,126.39,129.49$, 131.13, 133.70, 167.68, 168.56. FTMS+ESI calculated for $\mathrm{C}_{12} \mathrm{H}_{11} \mathrm{ClNO}_{3}[\mathrm{M}+\mathrm{H}]^{+}:$252.0427. Found: 252.0423.

\subsubsection{Synthesis of 1-acetyl-5-iodo-1H-indol-3-yl acetate 7 c}

Synthesised from 2-[(carboxymethyl)amino]-5-iodobenzoic acid (5d, 0.47 mmoles, $150 \mathrm{mg})$, acetic anhydride (27.57 mmoles, $2.602 \mathrm{~mL})$ and triethylamine (1.4 mmoles, 195.6 $\mu \mathrm{L}$ ) according to the representative procedure described in 2.2.29. Extractions were carried out with ethyl acetate. The product was purified by column chromatography (using 4/1= petroleum ether/ethyl acetate). Yellow solid. Yield: 53\%. $R_{\mathrm{f}} 0.41$ (using 4/1 petroleum ether/ethyl acetate). m.p. $125-131^{\circ} \mathrm{C} .{ }^{1} \mathrm{H}$ NMR $\left(\mathrm{CDCl}{ }_{3}, 400 \mathrm{MHz}, \mathrm{ppm}\right) \delta$ $2.38\left(3 \mathrm{H}, \mathrm{s}, \mathrm{CH}_{3} \mathrm{COO}\right), 2.59\left(3 \mathrm{H}, \mathrm{s}, \mathrm{CH}_{3} \mathrm{CON}\right), 7.65(1 \mathrm{H}, \mathrm{dd}, J=1.5,9.0 \mathrm{~Hz}, \mathrm{Ar}-\mathrm{H}), 7.68(1 \mathrm{H}, \mathrm{s}, \mathrm{Ar}-\mathrm{H}), 7.88(1 \mathrm{H}, \mathrm{d}, J=1.5 \mathrm{~Hz}, \mathrm{Ar}-\mathrm{H}), 8.22(1 \mathrm{H}, \mathrm{d}, J=9.0 \mathrm{~Hz}, \mathrm{Ar}-\mathrm{H}) .{ }^{13} \mathrm{C}$ $\mathrm{NMR}\left(\mathrm{CDCl}_{3}, 100 \mathrm{MHz}, \mathrm{ppm}\right) \delta 21.02,23.85,87.74,113.99,118.52,125.69,126.45,132.03,133.27,134.73,167.68,168.64$. IR (thin film): 1751, 1701, 1592, 1563, 1444 $\mathrm{cm}^{-1}$. FTMS+ESI calculated for $\mathrm{C}_{12} \mathrm{H}_{11} \mathrm{INO}_{3}[\mathrm{M}+\mathrm{H}]^{+}:$343.9784. Found: 343.9778 .

\subsubsection{Synthesis of 1-acetyl-5-fluoro-1H-indol-3-yl acetate $7 d$}

Synthesised from 2-[(carboxymethyl)amino]-5-fluorobenzoic acid (5e, 0.58 mmoles, $123 \mathrm{mg})$, acetic anhydride (34.06 mmoles, $3.214 \mathrm{~mL})$ and triethylamine (1.73 mmoles, $241.6 \mu \mathrm{L}$ ) according to the representative procedure described in 2.2.29. Extractions were carried out with dichloromethane. The product was purified by column chromatography (using $3 / 2=$ petroleum ether/ethyl acetate). Light green solid. Yield: $49 \% . R_{\mathrm{f}} 0.58$ (using $3 / 2$ petroleum ether/ethyl acetate). m.p. $142-143^{\circ} \mathrm{C} .{ }^{1} \mathrm{H}$ NMR $\left(\mathrm{CDCl}_{3}, 400 \mathrm{MHz}, \mathrm{ppm}\right) \delta 2.38\left(3 \mathrm{H}, \mathrm{s}, \mathrm{CH}_{3} \mathrm{COO}\right), 2.60\left(3 \mathrm{H}, \mathrm{s}, \mathrm{CH}_{3} \mathrm{CON}\right), 7.11(1 \mathrm{H}, \mathrm{t}, J=9.0 \mathrm{~Hz}, \mathrm{Ar}-\mathrm{H}), 7.19(1 \mathrm{H}, \mathrm{d}, J=8.0 \mathrm{~Hz}, \mathrm{Ar}-\mathrm{H}), 7.75(1 \mathrm{H}, \mathrm{s}, \mathrm{Ar}-\mathrm{H}), 8.43(1 \mathrm{H}, \mathrm{d}, J=$ 
$4.5 \mathrm{~Hz}, \mathrm{Ar}-\mathrm{H}) .{ }^{13} \mathrm{C} \mathrm{NMR}\left(\mathrm{CDCl}_{3}, 100 \mathrm{MHz}, \mathrm{ppm}\right) \delta 21.02,23.67,103.37$ (d, $\left.J 25.0 \mathrm{~Hz}\right), 114.06$ (d, $\left.J 24.5 \mathrm{~Hz}\right), 114.80,118.09$ (d, $J$ 9.0 Hz), $124.56(\mathrm{~d}, J 10.0 \mathrm{~Hz}), 129.23$, $134.21(\mathrm{~d}, J 4.0 \mathrm{~Hz}), 159.6(\mathrm{~d}, J 240.5 \mathrm{~Hz}), 167.73,168.50$. IR (thin film): 1753, 1694, $1449 \mathrm{~cm}^{-1}$. FTMS+ESI calculated for C $12 \mathrm{H}_{11} \mathrm{FNO}_{3}$ [M+H] $]^{+}: 236.0723$. Found: 236.0718 .

\subsubsection{Synthesis of 1-acetyl-5,6-difluoro-1H-indol-3-yl acetate 7 e}

Synthesised from 2-[(carboxymethyl)amino]-4,5-difluorobenzoic acid (5f, 0.65 mmoles, $150 \mathrm{mg})$, acetic anhydride (38.31 mmoles, $3.614 \mathrm{~mL})$ and triethylamine (1.95 mmoles, $271.7 \mu \mathrm{L}$ ) according to the representative procedure described in 2.2.29. Extractions were carried out with dichloromethane. The product was purified by column chromatography (using 3/2=petroleum ether/ethyl acetate). Cream coloured solid. Yield: $71 \%$. $R_{\mathrm{f}} 0.63$ (using $3 / 2$ petroleum ether/ethyl acetate). m.p. $122-125^{\circ} \mathrm{C}$. ${ }^{1} \mathrm{H}$ NMR $\left(\mathrm{CDCl}_{3}, 400 \mathrm{MHz}, \mathrm{ppm}\right) \delta 2.38\left(3 \mathrm{H}, \mathrm{s}, \mathrm{CH}_{3} \mathrm{COO}\right), 2.60\left(3 \mathrm{H}, \mathrm{s}, \mathrm{CH}_{3} \mathrm{CON}\right), 7.28(1 \mathrm{H}, \mathrm{dd}, J=8.0,9.5 \mathrm{~Hz}, \mathrm{Ar}-\mathrm{H}), 7.74(1 \mathrm{H}, \mathrm{s}, \mathrm{Ar}-\mathrm{H}), 8.35(1 \mathrm{H}, \mathrm{dd}, J=7.0,11.0 \mathrm{~Hz}, \mathrm{Ar}-\mathrm{H}) .{ }^{13} \mathrm{C}$ NMR ( $\left.\mathrm{CDCl}_{3}, 100 \mathrm{MHz}, \mathrm{ppm}\right) \delta 21.00,23.56,104.90$ (d, $J$ 21.0 Hz), 106.07 (d, J 24.5 Hz), 114.24 (d, J 4.0 Hz), 119.25 (d, J 7.5 Hz), 127.87 (d, J 9.5 Hz), $133.94,148.33$ $(\mathrm{dd}, J$ 15.0, $243.5 \mathrm{~Hz}), 149.80(\mathrm{dd}, J 15.5,245.5 \mathrm{~Hz}), 167.61,168.54$. IR (thin film): 1754, 1706, 1609, $1468 \mathrm{~cm}^{-1} . \mathrm{FTMS}^{\mathrm{E}} \mathrm{ESI}$ calculated for $\mathrm{C}_{12} \mathrm{H}_{10} \mathrm{~F}_{2} \mathrm{NO}_{3}[\mathrm{M}+\mathrm{H}]^{+}$: 254.0629. Found: 254.0622 .

\subsubsection{Synthesis of 1-acetyl-6-(trifluoromethyl)-1H-indol-3-yl acetate $7 f$}

Synthesised from 2-[(carboxymethyl)amino]-4-trifluorobenzoic acid (5g, 0.76 mmoles, $200 \mathrm{mg})$, acetic anhydride (44.86 mmoles, $4.233 \mathrm{~mL})$ and triethylamine (2.28 mmoles, 318.1 $\mu \mathrm{L}$ ) according to the representative procedure described in 2.2.29. Extractions were carried out with ethyl acetate. The product was purified by preparative HPLC equipped with a PLRP-S $100 \AA 8 \mu \mathrm{m}$ column, flow of $50 \mathrm{~mL} / \mathrm{min}$, solvent system $3 / 2$ acetonitrile/formic acid (0.1\%) and wavelength of 210 nm. White solid. Yield: $68 \% . R_{\mathrm{f}} 0.63$ (using $3 / 2$ petroleum ether/ethyl acetate). m.p. $115-121^{\circ} \mathrm{C} .{ }^{1} \mathrm{H}$ NMR $\left(\mathrm{CDCl}_{3}, 400 \mathrm{MHz}, \mathrm{ppm}\right) \delta 2.40\left(3 \mathrm{H}, \mathrm{s}, \mathrm{CH}{ }_{3} \mathrm{COO}\right), 2.64\left(3 \mathrm{H}, \mathrm{s}, \mathrm{CH}{ }_{3} \mathrm{CON}\right), 7.55(1 \mathrm{H}, \mathrm{d}, J=$ $8.5 \mathrm{~Hz}, \mathrm{Ar}-\mathrm{H}), 7.64(1 \mathrm{H}, \mathrm{d}, J=8.5 \mathrm{~Hz}, \mathrm{Ar}-\mathrm{H}), 7.86(1 \mathrm{H}, \mathrm{s}, \mathrm{Ar}-\mathrm{H}), 8.80(1 \mathrm{H}, \mathrm{s}, \mathrm{Ar}-\mathrm{H}) .{ }^{13} \mathrm{C} \mathrm{NMR}\left(\mathrm{CDCl}_{3}, 100 \mathrm{MHz}, \mathrm{ppm}\right) \delta 21.03,23.83,114.36(\mathrm{dd}, J 3.5,8.0 \mathrm{~Hz}), 115.66$, 
118.01, 120.54 (dd, $J 3.5,7.0 \mathrm{~Hz}), 123.14,125.86$ (d, $J 2.0 \mathrm{~Hz}), 128.25$ (q, $J 32.0 \mathrm{~Hz}), 131.89,134.03,167.71,168.67$. IR (thin film): 1766, 1715, 1571, 1437, 824 cm ${ }^{-1}$. FTMS+ESI calculated for $\mathrm{C}_{13} \mathrm{H}_{11} \mathrm{~F}_{3} \mathrm{NO}_{3}[\mathrm{M}+\mathrm{H}]^{+}:$286.0691. Found: 286.0686.

\subsubsection{Synthesis of 1-acetyl-5-methyl-1H-indol-3-yl acetate $7 g$}

Synthesised from 2-[(carboxymethyl)amino]-5-methylbenzoic acid (5h, 0.72 mmoles, $150 \mathbf{m g})$, acetic anhydride (42.33 mmoles, $3.994 \mathrm{~mL})$ and triethylamine (2.15 mmoles, $300.2 \mu \mathrm{L}$ ) according to the representative procedure described in 2.2.29. Extractions were carried out with dichloromethane. The product was purified by column chromatography (using 4/1 dichloromethane/hexane). Pale yellow solid. Yield: $67 \% . R_{\mathrm{f}} 0.68$ (using 4/1=dichloromethane/hexane). m.p. $113-115^{\circ} \mathrm{C} .{ }^{1} \mathrm{H}$ NMR $(\mathrm{CDCl}$, 400 MHz, ppm) $\delta 2.37\left(3 \mathrm{H}, \mathrm{s}, \mathrm{CH}_{3} \mathrm{COO}\right), 2.45\left(3 \mathrm{H}, \mathrm{s}, \mathrm{CH}_{3} \mathrm{Ar}\right), 2.58\left(3 \mathrm{H}, \mathrm{s}, \mathrm{CH}_{3} \mathrm{CON}\right), 7.20(1 \mathrm{H}, \mathrm{d}, J=8.5 \mathrm{~Hz}, \mathrm{Ar}-\mathrm{H}), 7.31(1 \mathrm{H}, \mathrm{s}, \mathrm{Ar}-\mathrm{H}), 7.65(1 \mathrm{H}, \mathrm{s}, \mathrm{Ar}-\mathrm{H}), 8.31(1 \mathrm{H}, \mathrm{d}, J=6.0$ $\mathrm{Hz}, \mathrm{Ar}-\mathrm{H}) .{ }^{13} \mathrm{C} \mathrm{NMR}\left(\mathrm{CDCl}_{3}, 100 \mathrm{MHz}, \mathrm{ppm}\right) \delta 21.05,21.39,23.82,113.38,116.37,117.28,123.79,127.55,131.19,133.47,134.48,167.92,168.57 . \mathrm{IR}(\mathrm{th}$ in film): 1760, 1695, 1452, 1376, $820 \mathrm{~cm}^{-1}$. FTMS+ESI calculated for $\mathrm{C}_{13} \mathrm{H}_{14} \mathrm{NO}_{3}[\mathrm{M}+\mathrm{H}]^{+}:$232.0974. Found: 232.0968 .

\subsubsection{Synthesis of 1-acetyl-7-methyl-1H-indol-3-yl acetate $7 \mathrm{~h}$}

Synthesised from 2-[(carboxymethyl)amino]-3-methylbenzoic acid (5i, 0.54 mmoles, $112 \mathrm{mg}$ ), acetic anhydride (31.61 mmoles, $2.982 \mathrm{~mL})$ and triethylamine (1.61 mmoles, 224.2 $\mu \mathrm{L}$ ) according to the representative procedure described in 2.2.29. Extractions were carried out with dichloromethane. The product was purified by preparative HPLC equipped with a PLRP-S $100 \AA 8$ m column, flow of $30 \mathrm{~mL} / \mathrm{min}$, solvent system $3 / 2$ acetonitrile/formic acid $(0.1 \%)$ and wavelength of 210 and 254 nm. Light pink solid. Yield: $34 \% . R_{\mathrm{f}} 0.32$ (using 4/1 dichloromethane/petroleum ether). m.p. $72-74{ }^{\circ} \mathrm{C} .{ }^{1} \mathrm{H} \mathrm{NMR}\left(\mathrm{CDCl}_{3}, 400 \mathrm{MHz}, \mathrm{ppm}\right) \delta 2.37\left(3 \mathrm{H}, \mathrm{s}, \mathrm{CH}{ }_{3} \mathrm{COO}\right), 2.57\left(3 \mathrm{H}, \mathrm{s}, \mathrm{CH}_{3} \mathrm{Ar}\right), 2.62(3 \mathrm{H}$, s, $\left.\mathrm{CH}_{3} \mathrm{CON}\right), 7.18-7.25(2 \mathrm{H}, \mathrm{m}, \mathrm{Ar}-\mathrm{H}), 7.37(1 \mathrm{H}, \mathrm{d}, J=7.5 \mathrm{~Hz}, \mathrm{Ar}-\mathrm{H}), 7.69(1 \mathrm{H}, \mathrm{s}, \mathrm{Ar}-\mathrm{H}) .{ }^{13} \mathrm{C} \mathrm{NMR}(\mathrm{CDCl}, 100 \mathrm{MHz}, \mathrm{ppm}) \delta 21.08,22.74,24.42,114.52,114.96,124.15$, 125.33, 127.07, 129.31, 132.60, 134.32, 167.77, 167.94. IR (thin film): 1766, 1720, 1369, $776 \mathrm{~cm}^{-1}$. FTMS+ESI calculated for $\mathrm{C}_{13} \mathrm{H}_{14} \mathrm{NO}_{3}[\mathrm{M}+\mathrm{H}]^{+}: 232.0974$. Found: 232.0967.

\section{RESULTS AND DISCUSSION}


At the outset of the programme the optimisation of two different synthetic methods for the synthesis of 2-[(carboxymethyl)amino]benzoic acids, that are key intermediates en-route to the final indoxyl targets, was undertaken, and the general approach is illustrated in Scheme 1. Initially, the method reported by Guyen et al. [13] was used, with modifications, for the synthesis of 2-[(carboxymethyl)amino]benzoic acid and a range of further functionalised derivatives (Scheme 2, Table 1). Thus reaction of the 2aminobenzoic acids $\mathbf{4 a - 4 g}$ with chloroacetic acid in sodium hydroxide (2M) at $80^{\circ} \mathrm{C}$ for 3 days, led to isolation of the 2 -[(carboxymethyl)amino]benzoic acids $\mathbf{5 a - 5 g}$ in 5 $29 \%$ yield after purification by chromatography. Attempts to purify $\mathbf{5 h}$ with both column chromatography and a Reveleris flash system equipped with a C18 reverse phase column were unsuccessful.

Given the low yields afforded during this first step in the synthesis of the indoxyl targets, an alternative route to the 2-[(carboxymethyl)amino]benzoic acids $\mathbf{5}$ was sought, and the method reported by Choi et al. [15] was utilised with small modifications. Thus 2-aminobenzoic acids 4 were subjected to a reductive alkylation using ethyl glyoxylate, acetic acid and sodium cyanoborohydride, to afford the ester intermediates $\mathbf{6 b}-\mathbf{6} \mathbf{f}$ and $\mathbf{6 h}$ in $80-90 \%$ yield (Scheme 3 , Table 2 , step 1 ). After a base hydrolysis with sodium hydroxide $(1 \mathrm{M})$ and precipitation with hydrochloric acid (1M), the 2-[(carboxymethyl)amino]benzoic acids $\mathbf{5 b - 5 f}$ and $\mathbf{5 h}$ were furnished in $67-100 \%$ yield (Scheme 3, Table 2, step 2). The syntheses of 5-iodo-2-[(2-ethoxy-2-oxoethyl)amino]benzoic acid (6d), 4,5-difluoro-2-[(2-ethoxy-2-oxoethyl)amino]benzoic acid (6f), 2[(carboxymethyl)amino]-4-trifluorobenzoic acid (5g) and 2-[(carboxymethyl)amino]-3-methylbenzoic acid (5i) are reported for the first time.

With the key 2-[(carboxymethyl)amino]benzoic acid intermediates $\mathbf{5}$ in hand, the next and final step for entry to the diacetylindoxyl targets $\mathbf{1}$ and $\mathbf{7}$ involved cyclisation and decarboxylation of the 2-[(carboxymethyl)amino]benzoic acids. As a starting point, the method used by Rodríguez-Domínguez et al. [12] with modifications, was employed. Thus, compound 5a was heated at reflux for 5 hours with acetic anhydride and anhydrous sodium acetate (Scheme 4). After purification via column chromatography, 1acetyl-1H-indol-3-yl acetate (1) was isolated in 26\% yield. With the aim of improving the yield and encouraged by the work by Lai $e t$ al. [14] where 1 -acetyl-1 $H$-indol-3-yl acetate was furnished through microwave activation in two steps from 2-[(carboxymethyl)amino]benzoic acid, it was also decided to attempt to synthesise $\mathbf{1}$ from $\mathbf{5 a}$, using microwave irradiation. Thus compound 5a was subjected to microwave irradiation for four minutes at $80^{\circ} \mathrm{C}$ and initial power of $300 \mathrm{~W}$ in the presence of sodium carbonate, 
$\mathrm{H}_{2} \mathrm{O}$ and acetic anhydride. 1-Acetyl-1H-indol-3-yl acetate was furnished directly from 5a, in 35\% yield, after purification via column chromatography. Different conditions (Scheme 4, Table 3) were employed for the microwave activation of 5a to investigate whether this would lead to an increase in reaction yield. An increase in microwave reaction time reduced the reaction yield (entry 2, Table 3), however when the reaction time was reduced, a small increase in yield was noticed (entry 3 , Table 3 ). Changing the base to triethylamine in the absence of $\mathrm{H}_{2} \mathrm{O}$ led to isolation of 1-acetyl-1H-indol-3-yl acetate in $40 \%$ yield (entry 4 , Table 3). The synthesis of derivatives of 1 was pursued using these optimised conditions as illustrated in Scheme 5, Table 4. All derivatives were afforded in good to very good yields and all 1-acetyl-1H-indol-3-yl acetates except for $\mathbf{7 h}$, were obtained in higher yields compared to $\mathbf{1}$. The lower yield obtained for formation of $\mathbf{1}$ correlates with the formation of a number of by-products as evidenced by thin layer chromatographic analysis of its reaction mixture. Also, the low solubilities of the starting materials $\mathbf{5 a}$ and $\mathbf{5 i}$ in acetic anhydride and triethylamine are likely to have contributed to the lower yields obtained for 1 -acetyl-1H-indol-3-yl acetates $\mathbf{1}$ and $\mathbf{7 h}$, compared to $\mathbf{7 a - 7 g}$. All of the 1 -acetyl-1H-indol-3-yl acetates were purified via column chromatography, with the novel $\mathbf{7 f}$ and $\mathbf{7 h}$ derivatives being purified via preparative HPLC.

\section{CONCLUSION}

A range of 2-[(carboxymethyl)amino]benzoic acids $\mathbf{5}$, that are key intermediates for the synthesis of the indoxyl targets $\mathbf{1}$ and $\mathbf{7}$, were synthesised using two different methods. The synthetic method to the benzoic acids $\mathbf{5}$ via intermediates $\mathbf{6}$, was found to be superior to the direct entry method from 2 -aminobenzoic acids $\mathbf{4}$ (summarised in Scheme 6) both in terms of yield and purification. Optimisation of the final step, by using microwave irradiation, and altering the base, solvent and reaction time, allowed rapid entry to a range of indoxyl derivatives $\mathbf{1}$ and $\mathbf{7}$ in good yield. Thus, an efficient method for the synthesis of 1 -acetyl-1H-indol-3-yl acetates, including novel derivatives, from 2-[(carboxymethyl)amino]benzoic acids has been developed.

\section{LIST OF ABBREVIATIONS}

$\mathrm{Ar}=$ Aromatic

${ }^{13} \mathrm{C}$ NMR = Carbon Nuclear Magnetic Resonance 
${ }^{1} \mathrm{H}$ NMR $=$ Proton Nuclear Magnetic Resonance

$\mathrm{CV}=$ Column Volume

Equiv. $=$ Equivalent

$\mathrm{ESI}=$ Electron Spray Ionisation

FTMS $=$ Fourier Transform Mass Spectrometry

HPLC = High Performance Liquid Chromatography

$\mathrm{MHz}=$ Megahertz

m.p. = Melting Point

$\mu \mathrm{L}=$ Microlitre

$\mu \mathrm{M}=$ Micrometer

$\mathrm{mL}=$ Millilitre

mmoles $=$ Millimoles

$\mathrm{M}=$ Molar

MW = Microwave

$\mathrm{nM}=$ Nanometer 
$\mathrm{rt}=$ room temperature

$\mathrm{UV}=$ Ultraviolet

\section{CONFLICT OF INTEREST}

The authors confirm that this article content has no conflict of interest.

\section{ACKNOWLEDGEMENTS}

We are grateful to the BBSRC (BB/HO15825/1) and Thermofisher Scientific for financial support. We are also grateful to Dr. A. J. André Cobb for provision of the microwave reactor, and to the University of Reading's Chemical Analysis Facility for access to the analytical instruments and technical support.

\section{SUPPORTING INFORMATION}

Supplementary data including spectroscopic data for this article can be accessed by contacting the corresponding author.

\section{REFERENCES}

[1] Buemi, M.R.; De Luca, L.; Chimirri, A.; Ferro, S.; Gitto, R.; Alvarez-Builla, J.; Alajarin, R. Indole derivatives as dual-effective agents for the treatment of neurodegenerative diseases: Synthesis, biological evaluation, and molecular modelling studies. Bioorg. Med. Chem., 2013, 21, 4575-4580.

[2] Silveira, C.C.; Mendes, S.R.; Soares, J.R.; Victoria, F.N.; Martinez, D.M. Synthesis and antioxidant activity of new C-3 sulfenyl indoles. Tetrahedron Lett., 2013, 54, 4926-4929. 
[3] Talaz, O.; Gülçin, I.; Göksu, S.; Saracoglu, N. Antioxidant activity of 5,10-dihydroindeno[1,2-b]indoles containing substituents on dihydroindeno part. Bioorg. Med. Chem., 2009, 17, 6583-6589.

[4] Sashidara, K.V.; Kumar, M.; Sonkar, R.; Singh, B.S.; Khanna, A.K.; Bhatia, G. Indole-based fibrates as potential hypolipidemic antiobesity agents. J. Med. Chem., $\mathbf{2 0 1 2}, 55,2769-2779$.

[5] Chang, H.; Wang, M.; Chan, M.; Chiu, Y.; Chen, Y. Antiobesity activities of indole-3-carbinol in high-fat-diet-induced obese mice. Nutrition, 2011, 27, 463-470.

[6] Agarwal, A.; Srivastava, K.; Puri, S.K.; Chauhan, P.M.S. Synthesis of substituted indole derivatives as a new class of antimalarial agents. Bioorg. Med. Chem. Lett., 2005, 15, 3133-3136.

[7] Kgokong, J.L.; Smith, P.P.; Matsabisa, G.M. 1,2,4-Triazino-[5,6b]indole derivatives: effects of the trifluoromethyl group on in vitro antimalarial activity. Bioorg. Med. Chem. Lett., 2005, 13, 2935-2942.

[8] Winter, C.A.; Risley, E.A., Nuss, G.W. Anti-inflammatory and antipyretic activities of indomethacin, 1-(p-chlorobenzoyl)-5-methoxy-2-methyl-indole-3-acetic acid. J. Pharmcol. Exp. Ther., 1963, 141, 369-376.

[9] Oliva, B.; Miller, K.; Caggiano, N.; O’Neill, A.J.; Cuny, G.D.; Hoemann, M.Z.; Hauske, J.R.; Chopra, I. Biological properties of novel antistaphylococcal quinoloneindole agents. Antimicrob. Agents Chemother., 2003, 47(2), 458-466.

[10] Berlin, W.; Sauer, B. In situ color detection of alpha-L-arabinofuranosidase, a "no-background" reporter gene, with 5-bromo-3-indolyl-alpha-L-arabinofuranoside. Anal. Biochem. 1996, 243, 171-5.

[11] Bovill, R.; Druggan, P. The use of chromogenic enzyme substrates in microbial identification. Culture, 2005, 26, 2, 5-8.

[12] Rodríguez-Domínguez, J.C.; Balbuzano-Deus, A.; López-López, M.A.; Kirsch, G. An improved synthesis of 1-acetyl-1H-indol-3-yl acetates. J. Heterocyclic Chem., 2007, 44, 273-275. 
[13] Guyen, B.; Schultes, C.M.; Hazel, P.; Mann, J.; Neidle, S. Synthesis and evaluation of analogues of 10H-indolo[3,2-b]quinolone as G-quadruplex stabilising ligands and potential inhibitors of the enzyme telomerase. Org. Biomol. Chem., 2004, 2, 981-988.

[14] Lai, T.K.; Chatterjee, A.; Banerji, J.; Sarkar, D.; Chattopadhyay, N. A concise synthesis of the DNA-intercalating and antimalarial alkaloid cryptolepine and its fluorescence behavior in solvents of different polarities. Helv. Chim. Acta, 2008, 91, 1975-1983.

[15] Choi, S.; Lee, J.; Jeong, S.; Im, I.; Lee, S.; Lee, E.; Lee, S.K.; Kwon, S.; Ahn, S.; Yoon, J.; Han, S.; Kim, J.; Kim, Y. 5,5'-Substituted indirubin-3'-oxime derivatives as potent cyclin-dependent kinase inhibitors with anticancer activity. J. Med. Chem., 2010, 53, 3696-3706.

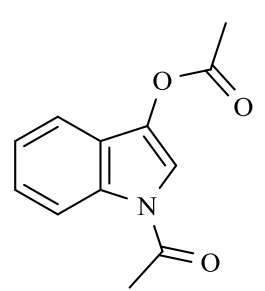

1-Acetyl-1 $H$-indol-3-yl acetate, 1

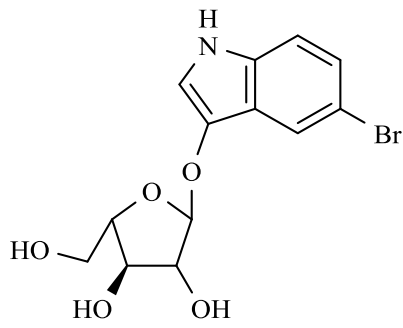

2

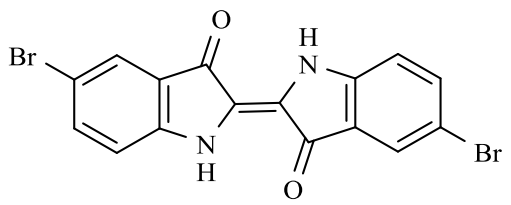

Figure 1. Structures of 1-acetyl-1H-indol-3-yl acetate and indoles of microbiological importance. 


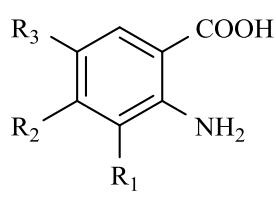

2-Aminobenzoic acids

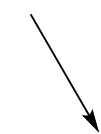

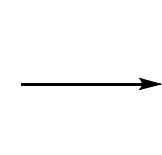

2-[(Carboxymethyl)amino]benzoic acids

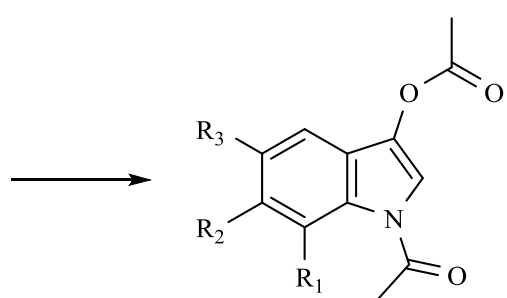

Indoxyl targets

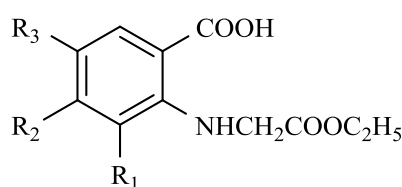

Scheme 1. Summary of the approaches investigated herein for the synthesis of the indoxyl targets.

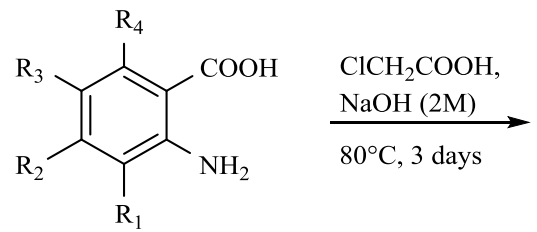

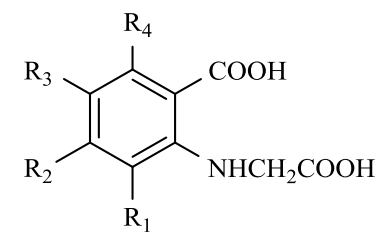

\begin{tabular}{|c|c|c|}
\hline Starting material & Product & Yield (\%) \\
\hline \hline 4a, $\mathrm{R}_{1,2,3,4}=\mathrm{H}$ & $\mathbf{5 a}, \mathrm{R}_{1,2,3,4}=\mathrm{H}$ & 29 \\
\hline
\end{tabular}




\begin{tabular}{|c|c|c|}
\hline $\mathbf{4 b}, \mathrm{R}_{1,2,4}=\mathrm{H}, \mathrm{R}_{3}=\mathrm{Br}$ & $\mathbf{5 b}, \mathrm{R}_{1,2,4}=\mathrm{H}, \mathrm{R}_{3}=\mathrm{Br}$ & 8 \\
\hline $\mathbf{4 c}, \mathrm{R}_{1,2,4}=\mathrm{H}, \mathrm{R}_{3}=\mathrm{Cl}$ & $\mathbf{5 c}, \mathrm{R}_{1,2,4}=\mathrm{H}, \mathrm{R}_{3}=\mathrm{Cl}$ & 24 \\
\hline $\mathbf{4 d}, \mathrm{R}_{1,2,4}=\mathrm{H}, \mathrm{R}_{3}=\mathrm{I}$ & $\mathbf{5 d}, \mathrm{R}_{1,2,4}=\mathrm{H}, \mathrm{R}_{3}=\mathrm{I}$ & 9 \\
\hline $\mathbf{4 e}, \mathrm{R}_{1,2,4}=\mathrm{H}, \mathrm{R}_{3}=\mathrm{F}$ & 5e, $\mathrm{R}_{1,2,4}=\mathrm{H}, \mathrm{R}_{3}=\mathrm{F}$ & 18 \\
\hline $\mathbf{4 f}, \mathrm{R}_{1,4}=\mathrm{H}, \mathrm{R}_{2,3}=\mathrm{F}$ & $\mathbf{5 f}, \mathrm{R}_{1,4}=\mathrm{H}, \mathrm{R}_{2,3}=\mathrm{F}$ & 5 \\
\hline $\mathbf{4 g}, \mathrm{R}_{1,3,4}=\mathrm{H}, \mathrm{R}_{2}=\mathrm{CF}_{3}$ & $\mathbf{5 g}, \mathrm{R}_{1,3,4}=\mathrm{H}, \mathrm{R}_{2}=\mathrm{CF}_{3}$ & 13 \\
\hline $\mathbf{4 h}, \mathrm{R}_{1,2,4}=\mathrm{H}, \mathrm{R}_{3}=\mathrm{CH}_{3}$ & $\mathbf{5 h}, \mathrm{R}_{1,2,4}=\mathrm{H}, \mathrm{R}_{3}=\mathrm{CH}_{3}$ & Not obtained pure \\
\hline
\end{tabular}

Scheme 2, Table 1. Synthesis of 2-[(carboxymethyl)amino]benzoic acids.

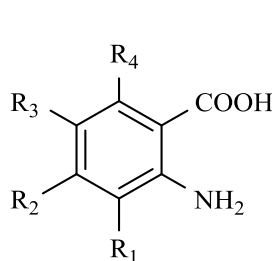

i) Ethyl glyoxylate

$\mathrm{CH}_{3} \mathrm{COOH}$

$\mathrm{MeOH}$

rt, 2.25 hours

ii) $\mathrm{NaBH}_{3}(\mathrm{CN})$

rt, 1.75 hours

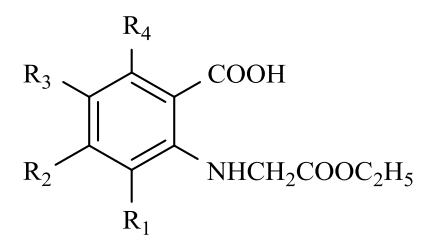

6

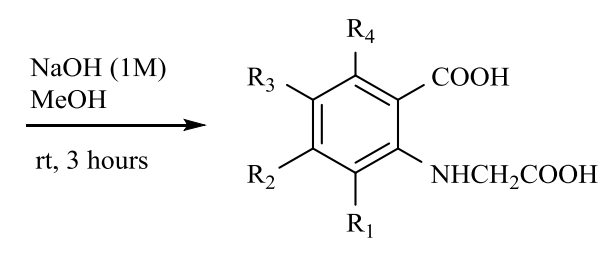

5 


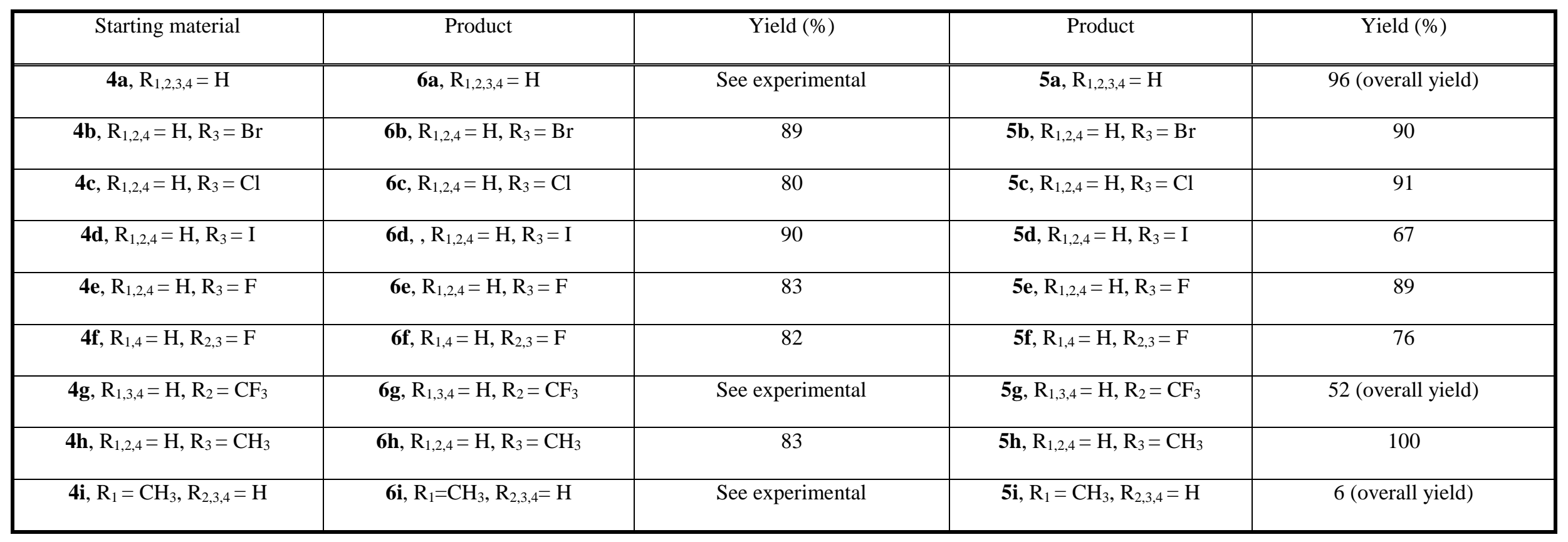

Scheme 3, Table 2. Synthesis of 2-[(carboxymethyl)amino]benzoic acids. 


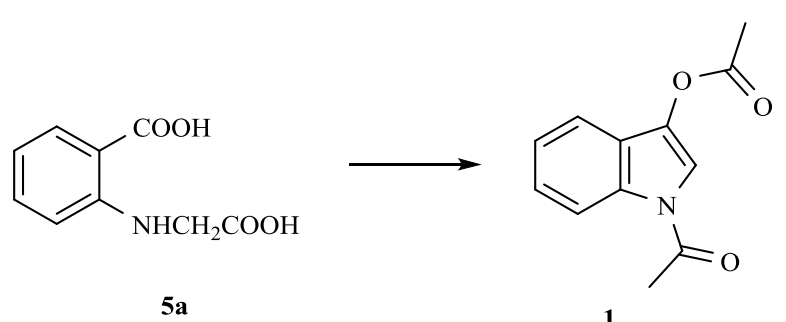

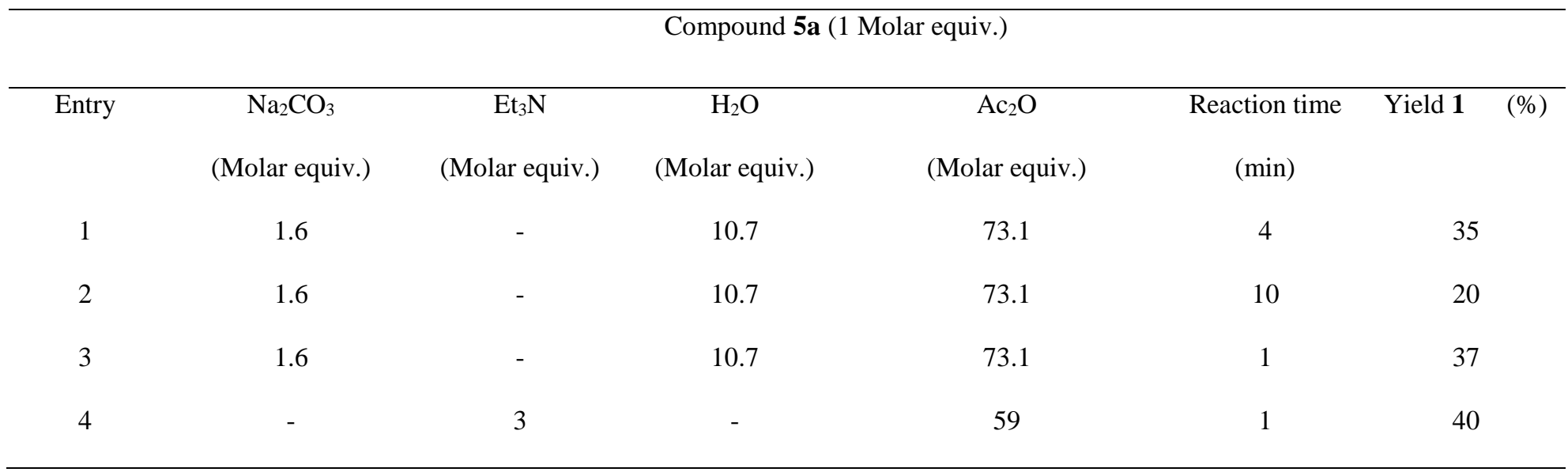

Scheme 4, Table 3. Summary of yields (\%) obtained during optimisation process. All reactions were carried out under microwave irradiation at $80{ }^{\circ} \mathrm{C}$ and initial power of $300 \mathrm{~W}$ with stirring. 


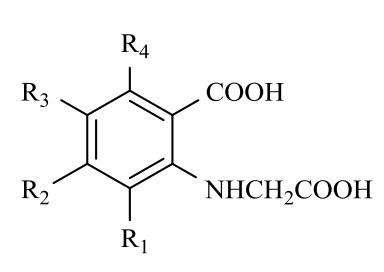

5

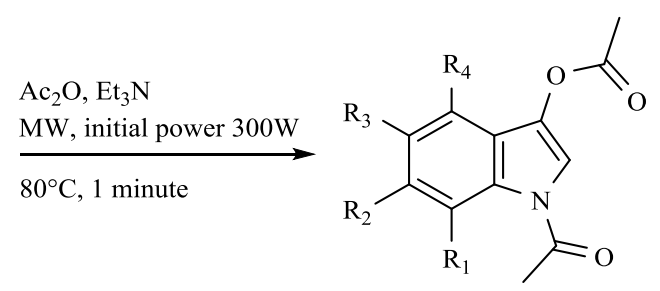

1 or 7

\begin{tabular}{|c|c|c|}
\hline Starting material & Product & Yield $(\%)$ \\
\hline$\overline{\mathbf{5 a}, \mathrm{R}_{1,2,3,4}=\mathrm{H}}$ & $\overline{\mathbf{1}, \mathrm{R}_{1,2,3,4}=\mathrm{H}}$ & 40 \\
\hline $\mathbf{5 b}, \mathrm{R}_{1,2,4}=\mathrm{H}, \mathrm{R}_{3}=\mathrm{Br}$ & $7 \mathbf{a}, \mathrm{R}_{1,2,4}=\mathrm{H}, \mathrm{R}_{3}=\mathrm{Br}$ & 59 \\
\hline $\mathbf{5 c}, \mathrm{R}_{1,2,4}=\mathrm{H}, \mathrm{R}_{3}=\mathrm{Cl}$ & $\mathbf{7 b}, \mathrm{R}_{1,2,4}=\mathrm{H}, \mathrm{R}_{3}=\mathrm{Cl}$ & 61 \\
\hline $\mathbf{5 d}, \mathrm{R}_{1,2,4}=\mathrm{H}, \mathrm{R}_{3}=\mathrm{I}$ & $\mathbf{7 c}, \mathrm{R}_{1,2,4}=\mathrm{H}, \mathrm{R}_{3}=\mathrm{I}$ & 53 \\
\hline 5e, $\mathrm{R}_{1,2,4}=\mathrm{H}, \mathrm{R}_{3}=\mathrm{F}$ & 7d, $R_{1,2,4}=H, R_{3}=F$ & 49 \\
\hline 5f, $\mathrm{R}_{1,4}=\mathrm{H}, \mathrm{R}_{2,3}=\mathrm{F}$ & $\mathbf{7 e}, \mathrm{R}_{1,4}=\mathrm{H}, \mathrm{R}_{2,3}=\mathrm{F}$ & 71 \\
\hline $\mathbf{5 g}, \mathrm{R}_{1,3,4}=\mathrm{H}, \mathrm{R}_{2}=\mathrm{CF}_{3}$ & $\mathbf{7 f}, \mathrm{R}_{1,3,4}=\mathrm{H}, \mathrm{R}_{2}=\mathrm{CF}_{3}$ & 68 \\
\hline $\mathbf{5 h}, \mathrm{R}_{1,2,4}=\mathrm{H}, \mathrm{R}_{3}=\mathrm{CH}_{3}$ & $\mathbf{7 g}, \mathrm{R}_{1,2,4}=\mathrm{H}, \mathrm{R}_{3}=\mathrm{CH}_{3}$ & 67 \\
\hline $\mathbf{5 i}, \mathrm{R}_{1}=\mathrm{CH}_{3}, \mathrm{R}_{2,3,4}=\mathrm{H}$ & $\mathbf{7 h}, \mathrm{R}_{1}=\mathrm{CH}_{3}, \mathrm{R}_{2,3,4}=\mathrm{H}$ & 34 \\
\hline
\end{tabular}

Scheme 5, Table 4. Synthesis of 1-acetyl-1H-indol-3-yl acetates. Reagents and conditions: Acetic anhydride, triethylamine, microwave, initial power $300 \mathrm{~W}, 80{ }^{\circ} \mathrm{C}, 1$ min. 


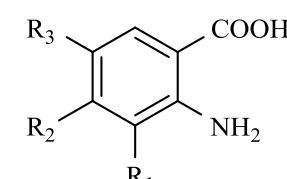

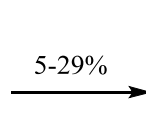

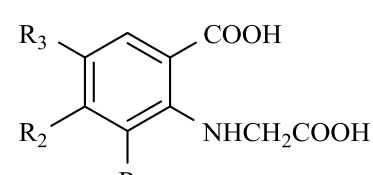

$\mathrm{R}_{1}$
MW,

initial power $300 \mathrm{~W}$,

$1 \mathrm{~min}, 80^{\circ} \mathrm{C}$

$34-71 \%$

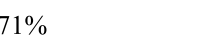

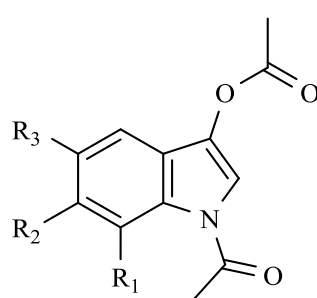

1 or 7

Scheme 6. Summary of the approaches investigated in this programme. 